\title{
On the Generalized Spectral Characterizations of Eulerian Graphs
}

\author{
Lihong Qiu \\ School of Mathematics and Statistics \\ Xi'an Jiaotong University \\ Xi'an, China \\ qiulihong20110126. com
}

\author{
Yizhe Ji Wei Wang* \\ School of Mathematics and Statistics \\ Xi'an Jiaotong University \\ Xi'an, China \\ \{yizheji, wang_weiw\}@xjtu.edu.cn
}

Submitted: Oct 22, 2018; Accepted: Jan 9, 2019; Published: Jan 25, 2019

(C) The authors. Released under the CC BY-ND license (International 4.0).

\begin{abstract}
A graph $G$ is said to be determined by its generalized spectra (DGS for short) if, for any graph $H$, graphs $H$ and $G$ are cospectral with cospectral complements imply that $H$ is isomorphic to $G$. In Wang [16] (J. Combin. Theory, Ser. B, 122 (2017) 438-451), the author gave a simple method for a graph to be DGS. However, the method does not apply to Eulerian graphs. In this paper, we give a simple method for a large family of Eulerian graphs to be DGS. Numerical experiments are also presented to illustrate the effectiveness of the proposed method.
\end{abstract}

Mathematics Subject Classifications: 05C50

\section{Introduction}

The spectrum of a graph encodes a lot of combinatorial information about the given graph and thus has long been a useful tool in spectral graph theory.

A fundamental question in this area is: "Which graphs are determined by their spectra (DS for short)?". The problem was first raised in 1956 by Günthard and Primas [8], which relates the theory of graph spectra to Hückel's theory [9] from chemistry. It is also closely related to a famous question of Kac [10]: "Can one hear the shape of a drum?". Fisher [6] modelled the drum by a graph, and the frequency of the sound was characterized by the eigenvalues of the graph. Thus, the two problems are essentially the same.

Another motivation for the above question comes from complexity theory. It is a long standing open question whether the graph isomorphism problem is an easy or a hard problem, despite the recent breakthrough result of Babai [1]. Since the spectrum can be computed in polynomial time, the focus is checking isomorphism for cospectral graphs.

\footnotetext{
*The corresponding author. Supported by National Science Foundation of China (11471005).
} 
However, as is generally known, proving graphs to be DS is more challenging than constructing cospectral graphs. Up to now, the techniques for proving DS graphs heavily depend on some special properties of the spectra of these graphs, which cannot be extended to general graphs. For the background and some known results about this problem, we refer the reader to $[4,5]$ and the references therein.

In recent years, Wang and $\mathrm{Xu}[13,14]$ and Wang $[15,16]$ considered the above problem in the context of the generalized spectra. A graph $G$ is determined by its generalized spectra (DGS for short) if, for any graph $H$, graphs $H$ and $G$ are cospectral with cospectral complements imply that $H$ is isomorphic to $G$. In Wang $[15,16]$, the author gave a simple method for determining whether a graph $G$ is DGS, which works for a large family of general graphs. To describe the result, let $G$ be a graph on $n$ vertices with adjacency matrix $A$. The walk-matrix of $G$ is defined as $W=W(G):=\left[e, A e, \cdots, A^{n-1} e\right]$ ( $e$ is the all-one vector). In $[15,16]$, Wang proved the following

Theorem 1 (Wang $[15,16])$. If $\operatorname{det} W(G) / 2^{\lfloor n / 2\rfloor}$ (which is always an integer) is odd and square-free, then $G$ is $D G S$.

It is noticed, however, the above theorem fails for Eulerian graphs. (Recall that a graph is Eulerian if it admits an Eulerian tour, which traverses each edge exactly once; or equivalently, if it is connected and the degree of every vertex is even). This is because for an Eulerian graph, every entry (except for the ones in the first column) of the walk-matrix is divisible by 2 , and hence $2^{n-1}$ divides $\operatorname{det} W$ and $\operatorname{det} W / 2^{\lfloor n / 2\rfloor}$ can never be odd and square-free.

This paper is devoted to investigating whether an Eulerian graph is DGS. The main contributions of the paper are as follows:

- We show that for an Eulerian graph $G$ with $\operatorname{det} W(G) / 2^{\left\lfloor\frac{3 n-3}{2}\right\rfloor}$ being odd and squarefree, $G$ is either DGS or there exists a regular rational orthogonal matrix $Q$ with level two (see Section 2 for the definitions) such that $Q^{T} A(G) Q$ is a $(0,1)$-matrix;

- Based on the above result, we give a simple sufficient condition for an Eulerian graph with the above property to be DGS, by constructing a digraph associated with $G$ and using a simple dimension argument.

It turns out that Eulerian graphs are among the most difficult family of graphs for which the existing method in $[15,16]$ does not work well. The main reason is that the exponent of 2 in the prime factorization of $\operatorname{det} W$ is usually too high for Eulerian graphs, and "excluding" the prime 2 constitutes the most difficult part in showing a graph $G$ to be DGS, as we shall see later.

We would like to mention that the proof of the main result of the paper follows the line of that in $[15,16]$. However, several new ingenious ideas are needed to make the proof work.

The rest of the paper is organized as follows. In Section 2, we give some preliminaries that will be needed later in the paper. In section 3, we show that for Eulerian graphs $G$ with $\operatorname{det} W(G) / 2^{\left\lfloor\frac{3 n-3}{2}\right\rfloor}$ being odd and square-free, the rational orthogonal matrices $Q$ 
such that $Q^{T} A(G) Q$ is (0,1)-matrix, must have level 1 or 2 . In Section 4 , an effective and novel method is provided to determine whether an Eulerian graph is DGS. In Section 5, we give some numerical results for illustrations. Conclusions and future work are given in Section 6.

\section{Preliminaries}

For convenience of the reader, we present some preliminary results that will be needed later in the paper.

Throughout, let $G=(V, E)$ be a simple graph with $(0,1)$-adjacency matrix $A=A(G)$. The spectrum of $G$ consists of all the eigenvalues (together with their multiplicities) of the matrix $A(G)$. The spectrum of $G$ together with that of its complement will be referred to as the generalized spectrum of $G$ in the paper (for some notions and terminologies in graph spectra, see e.g. [2]).

Two graphs are cospectral if they have the same spectrum. For a given graph $G$, we say that $G$ is determined by its spectrum (DS for short), if any graph having the same spectrum as $G$ is necessarily isomorphic to $G$. (Of course, the spectrum concerned should be specified.)

The walk-matrix of a graph $G$, denoted by $W(G)$ or simply $W$, is defined as

$$
\left[e, A e, A^{2} e, \cdots, A^{n-1} e\right]
$$

where $e$ denotes the all-one vector (we use this notation henceforth). There is a wellknown combinatorial interpretation of $W$, that is, the $(i, j)$-th entry of $W$ is the number of walks of $G$ starting from vertex $i$ with length $j-1$.

A graph $G$ is called controllable if $W$ is non-singular (see also [7]). Denoted by $\mathcal{G}_{n}$ the set of all controllable graphs on $n$ vertices. It was conjectured by Godsil that almost all graphs are controllable. Recently, O'Rourke and Touri [11] confirmed that the conjecture is true.

A rational orthogonal matrix $Q$ is an orthogonal matrix with all entries being rational numbers, and it is called regular if $Q e=e$.

The following theorem gives a simple characterization of cospectral graphs with respect to the generalized spectra.

Lemma 2 (c.f. Wang and Xu [13]). Suppose $G \in \mathcal{G}_{n}$. Then $G$ and $H$ are cospectral with respect to the generalized spectra if and only if there exists a unique regular rational orthogonal matrix $Q$ such that

$$
Q^{T} A(G) Q=A(H)
$$

Define

$$
\mathcal{Q}(G)=\left\{Q \in O_{n}(\mathbb{Q}) \mid Q^{T} A Q \text { is a }(0,1) \text {-matrix and } Q e=e\right\},
$$

where $O_{n}(\mathbb{Q})$ denotes the set of all orthogonal matrices with rational entries. 
Theorem 3 (c.f. Wang and $\mathrm{Xu}[13])$. Let $G \in \mathcal{G}_{n}$. Then $G$ is DGS if and only if the set $\mathcal{Q}(G)$ contains only permutation matrices.

By Theorem 3, in order to determine whether a given graph $G \in \mathcal{G}_{n}$ is DGS or not, we only need to check whether all the $Q$ 's in $\mathcal{Q}(G)$ are permutation matrices. For this purpose, the following definition is proved to be useful.

Definition 4. Let $Q$ be an orthogonal matrix with rational entries. The level of $Q$, denoted by $\ell(Q)$ or simply $\ell$, is the smallest positive integer $l$ such that $l Q$ is an integral matrix.

Clearly, $\ell$ is the least common denominator of all the entries of the matrix $Q$. If $\ell=1$, then $Q$ is a permutation matrix.

When dealing with integral and rational matrices, the Smith Normal Form (SNF for short) is a useful tool. An integral matrix $V$ of order $n$ is called unimodular if $\operatorname{det} V= \pm 1$. The following theorem is well-known.

Theorem 5 ([3]). For an integral matrix $M$, there exists unimodular matrices $V_{1}$ and $V_{2}$ such that $M=V_{1} S V_{2}$, where $S=\operatorname{diag}\left(d_{1}, d_{2}, \cdots, d_{n}\right)$ is the $\mathrm{SNF}$ of $M$ with $d_{i} \mid d_{i+1}$ for $i=1,2, \cdots, n-1 . d_{i}$ is called the $i$-th elementary divisor.

Note that the SNF of a matrix can be computed efficiently (see e.g. page 50 in [12]).

Theorem 6 (Wang and $\mathrm{Xu}[13])$. Let $W(G)$ be the walk-matrix of a graph $G \in \mathcal{G}_{n}$, and $Q \in \mathcal{Q}(G)$ with the level $\ell$. Then $\ell \mid d_{n}$, where $d_{n}$ is the $n$-th elementary divisor of the walk-matrix $W(G)$.

By the above theorem, $\ell$ is always a divisor of $d_{n}$, and hence is a divisor of $\operatorname{det} W$. However, the following theorem shows that not every divisor of $\operatorname{det} W$ can be a divisor of $\ell$.

Theorem 7 (Wang [15]). Let $G \in \mathcal{G}_{n}$ and $Q \in \mathcal{Q}(G)$ with level $\ell$. Let $p$ be an odd prime. If $p \mid \operatorname{det} W$ and $p^{2} \nmid \operatorname{det} W$, then $p$ cannot be a divisor of $\ell$.

By the above theorem, if $\operatorname{det} W= \pm 2^{m} b$ with $b$ being odd and square-free, then $\ell$ can only be a power of 2 . This fact will be heavily used in the sequel.

Now, we introduce the following family of Eulerian graphs which are the main focus of this paper:

$$
\Sigma_{n}=\left\{G \text { is an Eulerian graph of order } n \mid \operatorname{det} W(G) / 2^{\left\lfloor\frac{3 n-3}{2}\right\rfloor} \text { is odd and square-free }\right\} .
$$

We remark that $\operatorname{det} W(G) / 2^{\left\lfloor\frac{3 n-3}{2}\right\rfloor}$ is always an integer (see Corollary 17 in Section 3). By the definition of $\Sigma_{n}$, the exponent of 2 is much higher in the prime factorization of $\operatorname{det} W(G)$ than that in $[15,16]$, and unfortunately, all the previous methods cannot be applied to this situation, therefore we need to deal with the case from a new perspective.

Notations: We shall use the finite field notation $\mathbb{F}_{p}$ and $\bmod p$ (for a prime $p$ ) interchangeably, and shall use $\operatorname{rank}_{p}(M)$ to denote the rank of an integral $M$ over $\mathbb{F}_{p}$. 


\section{The level of $Q \in \mathcal{Q}(G)$ for $G \in \Sigma_{n}$}

By the previous discussions, we know that for a given graph $G$, in order to tell whether $G$ is DGS or not, it is crucial to determine the level of every regular rational orthogonal matrix $Q \in \mathcal{Q}(G)$. In this section, we show for any Eulerian graph $G \in \Sigma_{n}$, the level of $Q \in \mathcal{Q}(G)$ is very restrictive. The main result of this section is the following

Theorem 8. Let $G \in \Sigma_{n}$ be an Eulerian graph. Let $Q \in \mathcal{Q}(G)$ with level $\ell$. Then $\ell=1$ or 2 .

The proof of Theorem 8 is based on the following two theorems:

Theorem 9. Let $G \in \Sigma_{n}$. Then the SNF of $W$ is as follows:

$$
\operatorname{diag}(\underbrace{1,2, \cdots, 2}_{\left\lceil\frac{n+1}{2}\right\rceil}, \underbrace{2^{2}, 2^{2}, \cdots, 2^{2}, 2^{2} b}_{\left\lfloor\frac{n-1}{2}\right\rfloor}),
$$

where $b$ is an odd and square-free integer.

Theorem 10. Let $G \in \Sigma_{n}$. Let $Q \in \mathcal{Q}(G)$ with the level $\ell$, then $4 \nmid \ell$.

We postpone the proofs of the above theorems to the end of this section.

\subsection{A simple arithmetic property of Eulerian graphs}

In this subsection, we will present an arithmetic property about Eulerian graphs, which plays an important role in the sequel.

Lemma 11. Let $Q \in \mathcal{Q}(G)$ with level $\ell$. Suppose $\ell$ is even. Then there exists a $(0,1)$ vector $v \not \equiv 0(\bmod 2)$ such that

$$
v^{T} A^{k} v \equiv 0(\bmod 4), W^{T} v \equiv 0(\bmod 2),
$$

for any $k \geqslant 0$.

Proof. By definition, $Q \in \mathcal{Q}(G)$ implies that $Q^{T} A Q=B$ for some $(0,1)$-matrix $B$. Let $\bar{v}$ be the $i$-th column of $\ell Q$ such that $\bar{v} \not \equiv 0(\bmod 2)$ (such a $\bar{v}$ always exists by the definition of $\ell$ ). It follows from $Q^{T} A^{k} Q=B^{k}$ that $\bar{v}^{T} A^{k} \bar{v}=\ell^{2}\left(B^{k}\right)_{i, i} \equiv 0(\bmod 4)$. Let $v=\bar{v}+2 \beta$ be a $(0,1)$-vector for some integral vector $\beta$. Then

$$
v^{T} A^{k} v \equiv \bar{v}^{T} A^{k} \bar{v}+4 \bar{v} A^{k} \beta+4 \beta^{T} A^{k} \beta \equiv 0(\bmod 4) .
$$

The last assertion follows from the fact that $Q^{T} A^{k} Q=B^{k}$ and $Q e=e$ imply that $W^{T} Q$ is an integral matrix. Thus $W^{T} v \equiv 0(\bmod 2)$ holds.

Lemma 12. Let $Q \in \mathcal{Q}(G)$ with level $\ell \equiv 0(\bmod 4)$. Let $v=\left(v_{1}, v_{2}, \cdots, v_{n}\right)^{T}$ be any column of $\ell Q$. Suppose $m$ is the number of odd entries of $v$. Then $m \equiv 0(\bmod 4)$. 
Proof. Since $\ell \equiv 0(\bmod 4)$, we have $\ell=4 t$ for some integer $t$. Moreover, $v / \ell$ is a column of $Q$, it follows from the orthogonality of $Q$ that $(v / \ell)^{T}(v / \ell)=1$, i.e., $v^{T} v=\ell^{2}=16 t^{2}$, or equivalently, $v_{1}^{2}+v_{2}^{2}+\cdots+v_{n}^{2}=16 t^{2}$. For an odd entry $v_{i}$, we have $v_{i}=2 t_{i}+1$ for some integer $t_{i}$. It follows that $v_{i}^{2} \equiv 1(\bmod 4)$. Thus $v_{1}^{2}+v_{2}^{2}+\cdots+v_{n}^{2} \equiv m \equiv 0(\bmod 4)$.

The following lemma plays a significant role in establishing the main result in the paper.

Lemma 13. Let $G$ be an Eulerian graph and $Q \in \mathcal{Q}(G)$ with level $\ell \equiv 0(\bmod 4)$. Then there exists a $(0,1)$-vector $v \not \equiv 0(\bmod 2)$ such that

$$
W^{T} v \equiv 0(\bmod 4)
$$

Moreover, $v$ satisfies $v^{T} A^{k} v \equiv 0(\bmod 4)$ for any $k \geqslant 0$.

Proof. Let $\bar{v}$ be any column of $\ell Q$ with $\bar{v} \not \equiv 0(\bmod 4)$. Then we have $W^{T} \bar{v} \equiv 0(\bmod 4)$. Let $\bar{v}=v+2 u$, where $v$ is a $(0,1)$-vector and $u$ is an integral vector. Then $W^{T} \bar{v}=$ $W^{T}(v+2 u) \equiv 0(\bmod 4)$. i.e.,

$$
W^{T} u \equiv \frac{W^{T} v}{2}(\bmod 2)
$$

Note that $W=\left[e, A e, \cdots, A^{n-1} e\right]$. It follows that

$$
\left[u^{T} e, u^{T} A e, \cdots, u^{T} A^{n-1} e\right]^{T} \equiv\left[\frac{v^{T} e}{2}, \frac{v^{T} A e}{2}, \cdots, \frac{v^{T} A^{n-1} e}{2}\right]^{T}(\bmod 2) .
$$

Since $G$ is an Eulerian graph, we have $A e=\left[d_{1}, d_{2}, \cdots, d_{n}\right]^{T}$ with every $d_{i}$ being even, for $i=1,2, \cdots, n$. Therefore $u^{T} A^{i} e \equiv 0(\bmod 2)$, and hence $\frac{v^{T} A^{i} e}{2} \equiv 0(\bmod 2)$, for $i=1,2, \cdots, n-1$. In addition, the parity of $v$ is the same as that of $\bar{v}$. By Lemma 12, we have $v^{T} e=m \equiv 0(\bmod 4)$, where $m$ is the number of odd entries of $\bar{v}$. It follows that

$$
W^{T} v \equiv 0(\bmod 4)
$$

According to Lemma 11, $v$ satisfies $v^{T} A^{k} v \equiv 0(\bmod 4)$ for any $k=0,1, \cdots$. The proof is complete.

By Lemma 13, we introduce a new matrix

$$
\bar{W}:=\left[e, \frac{A e}{2}, \cdots, \frac{A^{n-1} e}{2}\right],
$$

which is clearly an integral matrix and satisfies $\bar{W}^{T} v \equiv 0(\bmod 2)$. The matrix $\bar{W}$ plays a similar role as that of $W(G)$ in $[16]$. 


\subsection{Some auxiliary lemmas}

In this subsection, we will present some lemmas, which are needed in the proof of the theorems in this section.

Lemma 14. Let $G$ be an Eulerian graph with adjacency matrix $A$. Then $e^{T} A^{2} e \equiv$ $0(\bmod 4)$, and $e^{T} A^{k} e \equiv 0(\bmod 8)$, for any integer $k \geqslant 3$,

Proof. First, we show $e^{T} A^{2} e \equiv 0(\bmod 4)$ holds. Let $d=\left(d_{1}, d_{2}, \cdots, d_{n}\right)^{T}$, where $d_{i}$ is the degree of the $i$-th vertex. Let $\hat{d}:=\frac{A e}{2}=\frac{d}{2}$. Then it is clear that $\hat{d}$ is an integral vector since every vertex degree is even in an Eulerian graph. It follows that $e^{T} A^{2} e=$ $(A e)^{T}(A e)=(2 \hat{d})^{T}(2 \hat{d})=4 \hat{d}^{T} \hat{d} \equiv 0(\bmod 4)$.

Next, we show $e^{T} A^{k} e \equiv 0(\bmod 8)$ for any $k \geqslant 3$.

Note that $e^{T} A^{k} e=(A e)^{T} A^{k-2}(A e)=(2 \hat{d})^{T} A^{k-2}(2 \hat{d})=4 \hat{d}^{T} A^{k-2} \hat{d}$. Let $l:=k-2$, then $e^{T} A^{k} e=4 \hat{d} A^{l} \hat{d}$. So it suffices to show $\hat{d}^{T} A^{l} \hat{d} \equiv 0(\bmod 2)$ for any $l \geqslant 1$. Next, we distinguish the following two cases:

Case 1. $l$ is even. It follows that

$$
\hat{d}^{T} A^{l} \hat{d}=\left(A^{\frac{l}{2}} \hat{d}\right)^{T}\left(A^{\frac{l}{2}} \hat{d}\right) \equiv \hat{d}^{T} A^{\frac{l}{2}} e=\hat{d}^{T} A^{\frac{l}{2}-1}(A e)=2 \hat{d}^{T} A^{\frac{l}{2}-1} \hat{d} \equiv 0(\bmod 2) .
$$

Case 2. $l$ is odd. Let $u:=A^{\frac{l-1}{2}} \hat{d}=\left(u_{1}, u_{2}, \cdots, u_{n}\right)^{T}$. It follows that

$$
\hat{d}^{T} A^{l} \hat{d}=\left(A^{\frac{l-1}{2}} \hat{d}\right)^{T} A\left(A^{\frac{l-1}{2}} \hat{d}\right)=\sum_{1 \leqslant i, j \leqslant n} a_{i, j} u_{i} u_{j}=2 \sum_{1 \leqslant i<j \leqslant n} a_{i, j} u_{i} u_{j} \equiv 0(\bmod 2) .
$$

Combining Cases 1 and 2, the proof is complete.

Lemma 15. Let $G$ be an Eulerian graph with adjacency matrix $A$. Then we have either $e^{T} A e \equiv 0(\bmod 4)$ or $e^{T} A e \equiv 2(\bmod 4)$. Moreover, if $e^{T} A e \equiv 0(\bmod 4)$, then $e^{T} A^{2} e \equiv$ $0(\bmod 8) ;$ otherwise if $e^{T} A e \equiv 2(\bmod 4)$, then $e^{T} A^{2} e \equiv 4(\bmod 8)$.

Proof. Note that $\hat{d}=\frac{A e}{2}=\left(\hat{d}_{1}, \hat{d}_{2}, \cdots, \hat{d}_{n}\right)^{T}$. If $e^{T} A e=2 \sum_{i=1}^{n} \hat{d}_{i} \equiv 0(\bmod 4)$, then it follows that $\sum_{i=1}^{n} \hat{d}_{i} \equiv 0(\bmod 2)$. Note $\sum_{i=1}^{n} \hat{d}_{i}^{2} \equiv \sum_{i=1}^{n} \hat{d}_{i} \equiv 0(\bmod 2)$. It follows that $e^{T} A^{2} e=(A e)^{T}(A e)=4 \sum_{i=1}^{n} \hat{d}_{i}^{2} \equiv 0(\bmod 8)$. Similarly, if $e^{T} A e=2 \sum_{i=1}^{n} \hat{d}_{i} \equiv 2(\bmod 4)$, then it follows that $\sum_{i=1}^{n} \hat{d}_{i} \equiv 1(\bmod 2)$. Note $\sum_{i=1}^{n} \hat{d}_{i}^{2} \equiv \sum_{i=1}^{n} \hat{d}_{i} \equiv 1(\bmod 2)$. It follows that $e^{T} A^{2} e=(A e)^{T}(A e)=4 \sum_{i=1}^{n} \hat{d}_{i}^{2} \equiv 4(\bmod 8)$. This completes the proof.

Lemma 16. Let $G$ be an Eulerian graph. Then $\operatorname{rank}_{2}(\bar{W}(G)) \leqslant\left\lceil\frac{n+1}{2}\right\rceil$. 
Proof. We shall prove the lemma in two cases: $n$ is even and $n$ is odd.

First suppose $n$ is even. Let $W_{1}$ be the matrix obtained from $\bar{W}$ by doubling the first two columns. Then it follows from Lemma 14 that

$$
\begin{aligned}
\bar{W}^{T} W_{1}= & {\left[\begin{array}{ccccc}
2 e^{T} e & e^{T} A e & \frac{e^{T} A^{2} e}{2} & \ldots & \frac{e^{T} A^{n-1} e}{2} \\
e^{T} A e & \frac{e^{T} A^{2} e}{2} & \frac{e^{T} A^{3} e}{4} & \ldots & \frac{e^{T} A^{n} e}{4} \\
e^{T} A^{2} e & \frac{e^{T} A^{3} e}{2} & \frac{e^{T} A^{4} e}{4} & \ldots & \frac{e^{T} A^{n+1} e}{4} \\
\vdots & \vdots & \vdots & \ddots & \vdots \\
e^{T} A^{n-1} e & \frac{e^{T} A^{n} e}{2} & \frac{e^{T} A^{n+1} e}{4} & \ldots & \frac{e^{T} A^{2 n-2} e}{4}
\end{array}\right] } \\
\equiv & {\left[\begin{array}{ccccc}
0 & 0 & 0 & \ldots & 0 \\
0 & 0 & 0 & \ldots & 0 \\
0 & 0 & 0 & \ldots & 0 \\
\vdots & \vdots & \vdots & \ddots & \vdots \\
0 & 0 & 0 & \ldots & 0
\end{array}\right](\bmod 2) . }
\end{aligned}
$$

It follows that $\operatorname{rank}_{2}(\bar{W})+\operatorname{rank}_{2}\left(W_{1}\right) \leqslant n$. Furthermore, $\operatorname{rank}_{2}\left(W_{1}\right) \geqslant \operatorname{rank}_{2}(\bar{W})-2$. Thus we have $\operatorname{rank}_{2}(\bar{W}) \leqslant \frac{n+2}{2}=\left\lceil\frac{n+1}{2}\right\rceil$.

Now suppose $n$ is odd. We further distinguish the following two cases:

Case 1. If $e^{T} A e \equiv 0(\bmod 4)$, then from Lemma 15 , we can get $e^{T} A^{2} e \equiv 0(\bmod 8)$. So it follows from Lemma 14 that

$$
\begin{aligned}
\bar{W}^{T} \bar{W} & =\left[\begin{array}{ccccc}
e^{T} e & \frac{e^{T} A e}{2} & \frac{e^{T} A^{2} e}{2} & \ldots & \frac{e^{T} A^{n-1} e}{2} \\
\frac{e^{T} A e}{2} & \frac{e^{T} A^{2} e}{4} & \frac{e^{T} A^{3} e}{4} & \ldots & \frac{e^{T} A^{n} e}{4} \\
\frac{e^{T} A^{2} e}{2} & \frac{e^{T} A^{3} e}{4} & \frac{e^{T} A^{4} e}{4} & \ldots & \frac{e^{T} A^{n+1} e}{4} \\
\vdots & \vdots & \vdots & \ddots & \vdots \\
\frac{e^{T} A^{n-1} e}{2} & \frac{e^{T} A^{n} e}{4} & \frac{e^{T} A^{n+1} e}{4} & \ldots & \frac{e^{T} A^{2 n-2} e}{4}
\end{array}\right] \\
& \equiv\left[\begin{array}{ccccc}
1 & 0 & 0 & \ldots & 0 \\
0 & 0 & 0 & \ldots & 0 \\
0 & 0 & 0 & \ldots & 0 \\
\vdots & \vdots & \vdots & \ddots & \vdots \\
0 & 0 & 0 & \ldots & 0
\end{array}\right](\bmod 2) .
\end{aligned}
$$

Note that $2 \operatorname{rank}_{2}(\bar{W})=\operatorname{rank}_{2}\left(\bar{W}^{T}\right)+\operatorname{rank}_{2}(\bar{W}) \leqslant n+\operatorname{rank}_{2}\left(\bar{W}^{T} \bar{W}\right)=n+1$. It follows that $\operatorname{rank}_{2}(\bar{W}) \leqslant \frac{n+1}{2}=\left\lceil\frac{n+1}{2}\right\rceil$.

Case 2. If $e^{T} A e \equiv 2(\bmod 4)$, then from Lemma 15 , we can get $e^{T} A^{2} e \equiv 4(\bmod 8)$. So 
it follows from Lemma 14 that

$$
\begin{aligned}
\bar{W}^{T} \bar{W} & =\left[\begin{array}{ccccc}
e^{T} e & \frac{e^{T} A e}{2} & \frac{e^{T} A^{2} e}{2} & \ldots & \frac{e^{T} A^{n-1} e}{2} \\
\frac{e^{T} A e}{2} & \frac{e^{T} A^{2} e}{4} & \frac{e^{T} A^{3} e}{4} & \ldots & \frac{e^{T} A^{n} e}{4} \\
\frac{e^{T} A^{2} e}{2} & \frac{e^{T} A^{3} e}{4} & \frac{e^{T} A^{4} e}{4} & \ldots & \frac{e^{T} A^{n+1} e}{4} \\
\vdots & \vdots & \vdots & \ddots & \vdots \\
\frac{e^{T} A^{n-1} e}{2} & \frac{e^{T} A^{n} e}{4} & \frac{e^{T} A^{n+1} e}{4} & \ldots & \frac{e^{T} A^{2 n-2} e}{4}
\end{array}\right] \\
& \equiv\left[\begin{array}{ccccc}
1 & 1 & 0 & \ldots & 0 \\
1 & 1 & 0 & \ldots & 0 \\
0 & 0 & 0 & \ldots & 0 \\
\vdots & \vdots & \vdots & \ddots & \vdots \\
0 & 0 & 0 & \ldots & 0
\end{array}\right](\bmod 2) .
\end{aligned}
$$

Similarly $2 \operatorname{rank}_{2}(\bar{W}) \leqslant n+\operatorname{rank}_{2}\left(\bar{W}^{T} \bar{W}\right)=n+1$. It follows that $\operatorname{rank}_{2}(\bar{W}) \leqslant \frac{n+1}{2}=$ $\left\lceil\frac{n+1}{2}\right\rceil$.

This completes the proof.

As a corollary, we have

Corollary 17. Let $G$ be an Eulerian graph. Then the exponent of 2 of in the prime factorization of $\operatorname{det} W$ is at least $\left\lfloor\frac{3 n-3}{2}\right\rfloor$.

Proof. Since $\operatorname{rank}_{2}(\bar{W}(G)) \leqslant\left\lceil\frac{n+1}{2}\right\rceil$, the number of even numbers in the diagonal entries of $\bar{W}$ is at least $n-\left\lceil\frac{n+1}{2}\right\rceil=\left\lfloor\frac{n-1}{2}\right\rfloor$. It follows from the definition of $\bar{W}$ that $2^{n-1+\left\lfloor\frac{n-1}{2}\right\rfloor}$ always divides $\operatorname{det} W$.

The following lemma gives the SNF of $\bar{W}$, the proof of which follows the same idea as Lemma 3.5 in [16].

Lemma 18. Let $G \in \Sigma_{n}$. Then $\operatorname{rank}_{2}(\bar{W})=\left\lceil\frac{n+1}{2}\right\rceil$ and the SNF of $\bar{W}$ is as follows:

$$
S=\operatorname{diag}(\underbrace{1,1, \cdots, 1}_{\left\lceil\frac{n+1}{2}\right\rceil}, \underbrace{2,2, \cdots, 2,2 b}_{\left\lfloor\frac{n-1}{2}\right\rfloor}),
$$

where $b$ is an odd square-free integer.

Proof. Since $\operatorname{det} W / 2^{2^{\left.\frac{3 n-3}{2}\right\rfloor}}$ is odd and square-free, we have $\operatorname{det} \bar{W}= \pm 2^{\left\lfloor\frac{n-1}{2}\right\rfloor} p_{1} p_{2} \cdots p_{s}$, where $p_{i}$ 's are distinct odd primes for each $i$. Thus the SNF of $\bar{W}$ can be written as $S=\operatorname{diag}\left(1,1, \ldots, 1,2^{l_{1}}, 2^{l_{2}}, \ldots, 2^{l_{t}} b\right)$, where $b=p_{1} p_{2} \ldots p_{s}$ is an odd square-free integer. It follows from Lemma 16 that $\operatorname{rank}_{2}(\bar{W}) \leqslant\left\lceil\frac{n+1}{2}\right\rceil$, i.e., $n-t \leqslant\left\lceil\frac{n+1}{2}\right\rceil$. Thus, we have $t \geqslant$ $n-\left\lceil\frac{n+1}{2}\right\rceil=\left\lfloor\frac{n-1}{2}\right\rfloor$. Moreover, we have $l_{1}+l_{2}+\ldots+l_{t}=\left\lfloor\frac{n-1}{2}\right\rfloor$, since $\operatorname{det}(\bar{W})= \pm \operatorname{det}(S)$. It follows that $l_{1}=l_{2}=\ldots=l_{t}=1$ and $t=\left\lfloor\frac{n-1}{2}\right\rfloor$. The proof is complete.

The following corollary says that if $G \in \Sigma_{n}$ is an Eulerian graph with an even number of vertices, then the number of edges of $G$ must be odd. 
Corollary 19. Let $G \in \Sigma_{n}$. If $n$ is even, then $e^{T} A e \equiv 2(\bmod 4)$.

Proof. We prove the corollary by contradiction. Suppose $e^{T} A e \equiv 0(\bmod 4)$. According to Lemma 15, we have $e^{T} A^{2} e \equiv 0(\bmod 8)$. Consequently,

$$
\begin{aligned}
\bar{W}^{T} \bar{W}= & {\left[\begin{array}{ccccc}
e^{T} e & \frac{e^{T} A e}{2} & \frac{e^{T} A^{2} e}{2} & \ldots & \frac{e^{T} A^{n-1} e}{2} \\
\frac{e^{T} A e}{2} & \frac{e^{T} A^{2} e}{4} & \frac{e^{T} A^{3} e}{4} & \ldots & \frac{e^{T} A^{n} e}{4} \\
\frac{e^{T} A^{2} e}{2} & \frac{e^{T} A^{3} e}{4} & \frac{e^{T} A^{4} e}{4} & \ldots & \frac{e^{T} A^{n} e}{4} \\
\vdots & \vdots & \vdots & \ddots & \vdots \\
\frac{e^{T} A^{n-1} e}{2} & \frac{e^{T} A^{n} e}{4} & \frac{e^{T} A^{n+1} e}{4} & \ldots & \frac{e^{T} A^{2 n-2} e}{4}
\end{array}\right] } \\
& \equiv\left[\begin{array}{ccccc}
0 & 0 & 0 & \ldots & 0 \\
0 & 0 & 0 & \ldots & 0 \\
0 & 0 & 0 & \ldots & 0 \\
\vdots & \vdots & \vdots & \ddots & \vdots \\
0 & 0 & 0 & \ldots & 0
\end{array}\right](\bmod 2) .
\end{aligned}
$$

It follows that $2 \operatorname{rank}_{2}(\bar{W})=\operatorname{rank}_{2}\left(\bar{W}^{T}\right)+\operatorname{rank}_{2}(\bar{W}) \leqslant n$, and hence $\operatorname{rank}_{2}(\bar{W}) \leqslant \frac{n}{2}$. By Lemma 18, we have $\operatorname{rank}_{2}(\bar{W})=\left\lceil\frac{n+1}{2}\right\rceil$. Since $n$ is even, we have $1+\frac{n}{2}=\left\lceil\frac{n+1}{2}\right\rceil \leqslant \frac{n}{2}$; a contradiction. This completes the proof.

For convenience, next, we fix some notations. Let $\hat{W}$ be the matrix defined as follows:

$$
\hat{W}= \begin{cases}{\left[\frac{A^{2} e}{2}, \frac{A^{3} e}{2}, \ldots, \frac{A^{\frac{n}{2}} e}{2}\right],} & \text { if } n \text { is even; } \\ {\left[\frac{A e}{2}, \frac{A^{2} e}{2}, \ldots, \frac{A^{\frac{n-1}{2}} e}{2}\right],} & \text { if } n \text { is odd and } e^{T} A e \equiv 0(\bmod 4) . \\ {\left[\frac{A e}{2}-e, \frac{A^{2} e}{2}, \ldots, \frac{A^{\frac{n-1}{2}} e}{2}\right],} & \text { if } n \text { is odd and } e^{T} A e \equiv 2(\bmod 4) .\end{cases}
$$

Lemma 20. Let $G \in \Sigma_{n}$. Then we have $\operatorname{rank}_{2}(\hat{W})=\left\lfloor\frac{n-1}{2}\right\rfloor$.

Proof. Since $G \in \Sigma_{n}$, we have $\operatorname{rank}_{2}(\bar{W})=\left\lceil\frac{n+1}{2}\right\rceil=: k$ according to Lemma 18. It suffices to show that the first $k$ columns of $\bar{W}$ are linearly independent over $\mathbb{F}_{2}$. For contradiction, suppose $e, \frac{A e}{2}, \cdots, \frac{A^{k-1} e}{2}$ are linearly dependent, i.e., there exist $c_{0}, c_{1}, \cdots, c_{k-1} \in \mathbb{F}_{2}$, not all zero, such that $c_{0} e+c_{1} \frac{A e}{2}+\cdots+c_{k-1} \frac{A^{k-1} e}{2}=0$. Let $m$ be the maximum index among $0,1, \cdots, k-1$ with $c_{m} \neq 0$. Then we have $0<m \leqslant k-1$ and

$$
\frac{A^{m} e}{2}=-c_{m}^{-1} c_{0} e-c_{m}^{-1} c_{1} \frac{A e}{2}-\cdots-c_{m}^{-1} c_{m-1} \frac{A^{m-1} e}{2} \text { over } \mathbb{F}_{2}
$$

i.e., $\frac{A^{m} e}{2} \in \operatorname{span}\left\{e, \frac{A e}{2}, \cdots, \frac{A^{m-1} e}{2}\right\}$. It follows from Eq. (11) that

$$
\frac{A^{m} e}{2}=-c_{m}^{-1} c_{0} e-c_{m}^{-1} c_{1} \frac{A e}{2}-\cdots-c_{m}^{-1} c_{m-1} \frac{A^{m-1} e}{2}+2 \beta \text { over } \mathbb{Z}
$$


for some integral vector $\beta$. Left-multiplying $A$ on both sides of Eq. (12) gives that

$$
\frac{A^{m+1} e}{2}=-2 c_{m}^{-1} c_{0} \frac{A e}{2}-c_{m}^{-1} c_{1} \frac{A^{2} e}{2}-\cdots-c_{m}^{-1} c_{m-1} \frac{A^{m} e}{2}+2 A \beta,
$$

i.e.,

$$
\frac{A^{m+1} e}{2}=-c_{m}^{-1} c_{1} \frac{A^{2} e}{2}-\cdots-c_{m}^{-1} c_{m-1} \frac{A^{m} e}{2} \text { over } \mathbb{F}_{2} .
$$

It follows that $\frac{A^{m+1} e}{2} \in \operatorname{span}\left\{e, \frac{A e}{2}, \cdots, \frac{A^{m-1} e}{2}\right\}$. Similarly, we have

$$
\frac{A^{m+t} e}{2} \in \operatorname{span}\left\{e, \frac{A e}{2}, \cdots, \frac{A^{m-1} e}{2}\right\},
$$

for any $t \geqslant 0$. Thus we have $\operatorname{rank}_{2}(\bar{W}) \leqslant m \leqslant k-1$; a contradiction. This completes the proof.

Lemma 21. Let $G$ be an Eulerian graph and $P_{G}(x)=x^{n}+c_{1} x^{n-1}+\cdots+c_{n-1} x+c_{n}$ the characteristic polynomial of graph $G$. Then $c_{n}$ is even. Moreover, define

$$
\tilde{W}:=\left[\frac{A e}{2}, \frac{A^{2} e}{2}, \cdots, \frac{A^{n-1} e}{2},-\frac{A^{n} e}{2}-\frac{c_{n}}{2} e+e\right] .
$$

Then $\operatorname{det}(\tilde{W})= \pm \operatorname{det}(\bar{W})$.

Proof. By Cayley-Hamilton's Theorem, we have

$$
A^{n}+c_{1} A^{n-1}+\cdots+c_{n-1} A+c_{n} I=0 .
$$

Right-multiplying the vector $e$ on both sides of Eq. (15) and using the fact $A^{i} e \equiv 0(\bmod 2)$ for $i=1,2, \cdots, n$ gives that $c_{n}$ is even. Thus we have

$$
\frac{A^{n} e}{2}+c_{1} \frac{A^{n-1} e}{2}+\cdots+c_{n-1} \frac{A e}{2}+\frac{c_{n}}{2} e=0,
$$

i.e.,

$$
c_{1} \frac{A^{n-1} e}{2}+\cdots+c_{n-1} \frac{A e}{2}=-\frac{A^{n} e}{2}-\frac{c_{n}}{2} e .
$$

Recall that $\bar{W}=\left[e, \frac{A e}{2}, \cdots, \frac{A^{n-1} e}{2}\right]$, By performing the elementary column operations to $\bar{W}$, it is easy to see that $\operatorname{det} \bar{W}=\operatorname{det}\left[-\frac{A^{n} e}{2}-\frac{c_{n}}{2} e+e, \frac{A e}{2}, \cdots, \frac{A^{n-1} e}{2}\right]$. Then the matrix $\tilde{W}$ can be obtained by switching the columns of $\bar{W}$. It is obvious that $\operatorname{det}(\tilde{W})= \pm \operatorname{det}(\bar{W})$. So the lemma follows and the proof is complete.

Let $W_{1}$ be the matrix obtained from the matrix $\bar{W}$ and defined as follows:

$$
W_{1}= \begin{cases}{\left[2 e, A e, \frac{A^{2} e}{2}, \ldots, \frac{A^{n-1} e}{2}\right],} & \text { if } n \text { is even; } \\ {\left[2 e, \frac{A e}{2}, \frac{A^{2} e}{2}, \ldots, \frac{A^{n-1} e}{2}\right],} & \text { if } n \text { is odd and } e^{T} A e \equiv 0(\bmod 4) ; \\ {\left[2 e, \frac{A e}{2}-e, \frac{A^{2} e}{2}-2 A e+2 e, \frac{A^{3} e}{2}, \ldots, \frac{A^{n-1} e}{2}\right],} & \text { if } n \text { is odd and } e^{T} A e \equiv 2(\bmod 4) .\end{cases}
$$


Similarly, $\hat{W}_{1}$ is defined as follows:

$$
\hat{W}_{1}= \begin{cases}{\left[\frac{A^{3} e}{2}, \frac{A^{5} e}{2}, \ldots, \frac{A^{n-1} e}{2}\right],} & \text { if } n \text { is even. } \\ {\left[\frac{A^{2} e}{2}, \frac{A^{4} e}{2}, \ldots, \frac{A^{n-1} e}{2}\right],} & \text { if } n \text { is odd and } e^{T} A e \equiv 0(\bmod 4) . \\ {\left[\frac{A^{2} e}{2}-2 A e+2 e, \frac{A^{4} e}{2}, \ldots, \frac{A^{n-1} e}{2}\right],} & \text { if } n \text { is odd and } e^{T} A e \equiv 2(\bmod 4) .\end{cases}
$$

Lemma 22. Let $G \in \Sigma_{n}$. If $n$ is even, then $\operatorname{rank}_{2}\left(\frac{\tilde{W}^{T} \hat{W}_{1}}{2}\right)=\left\lfloor\frac{n-1}{2}\right\rfloor$, where $\tilde{W}$ is defined as in Eq. (14). If $n$ is odd, then $\operatorname{rank}_{2}\left(\frac{\bar{W}^{T} \hat{W}_{1}}{2}\right)=\left\lfloor\frac{n-1}{2}\right\rfloor$.

Proof. Let $n$ be even. Let $W_{1}$ be defined as the above. It is easy to verify that $\frac{\tilde{W}^{T} W_{1}}{2}$ is an integral matrix. By Lemma 18 and Lemma 21, it can be computed that

$$
\operatorname{det}\left(\frac{\tilde{W}^{T} W_{1}}{2}\right)= \pm\left(2^{\left\lfloor\frac{n-1}{2}\right\rfloor} b\right)^{2} \cdot 2^{2} / 2^{n}= \pm b^{2} .
$$

Therefore, the column vectors of matrix $\frac{\tilde{W}^{T} W_{1}}{2}$ are linearly independent, over $\mathbb{F}_{2}$. It follows that $\operatorname{rank}_{2}\left(\frac{\tilde{W}^{T} \hat{W}_{1}}{2}\right)$ equals the number of columns of $\hat{W}_{1}$, which is $\frac{n-2}{2}=\left\lfloor\frac{n-1}{2}\right\rfloor$.

Let $n$ be odd. By the definitions of $\bar{W}$ and $W_{1}$, it is easy to see that $\frac{\bar{W}^{T} W_{1}}{2}$ is an integral matrix. By Lemma 18, it can be computed that



Therefore, the column vectors of matrix $\frac{\bar{W}^{T} W_{1}}{2}$ are linearly independent, over $\mathbb{F}_{2}$. Thus, $\operatorname{rank}_{2}\left(\frac{\bar{W}^{T} \hat{W}_{1}}{2}\right)$ equals the number of columns of $\hat{W}_{1}$, which is $\frac{n-1}{2}=\left\lfloor\frac{n-1}{2}\right\rfloor$.

\subsection{Proof of Theorem 10}

Now we are ready to present the proof of Theorem 10:

Proof. We prove the theorem by contradiction. Suppose on the contrary that $4 \mid \ell$. It follows from Lemma 11 and Lemma 13 that there exists a vector $v \not \equiv 0(\bmod 2)$ such that $v^{T} A^{k} v \equiv 0(\bmod 4)$ for any $k \geqslant 0$ and $\bar{W}^{T} v \equiv 0(\bmod 2)$. Note that $v$ is a solution to the system of linear equations $\bar{W}^{T} v \equiv 0(\bmod 2)$. Note that $G \in \Sigma_{n}$. It follows from Lemma 18 that $\operatorname{rank}_{2}(\overline{\mathrm{W}})=\left\lceil\frac{\mathrm{n}+1}{2}\right\rceil$. According to Lemma 20, we can obtain that $\operatorname{rank}_{2}(\hat{W})=\left\lfloor\frac{n-1}{2}\right\rfloor$. Moreover, it is not difficult to verify that $\bar{W}^{T} \hat{W} \equiv 0(\bmod 2)$. Since $\operatorname{rank}_{2}(\overline{\mathrm{W}})=\left\lceil\frac{\mathrm{n}+1}{2}\right\rceil$, the dimension of the solution space of the system of linear equations $\bar{W}^{T} x \equiv 0(\bmod 2)$ is $\left\lfloor\frac{n-1}{2}\right\rfloor$, which is equal to $\operatorname{rank}_{2}(\hat{W})$. Therefore, the column vectors of $\hat{W}$ can be chosen as a basis of the solution space of $\bar{W}^{T} x \equiv 0(\bmod 2)$. It follows 
that $v$ can be written as the linear combination of the column vectors of $\hat{W}$ over $\mathbb{F}_{2}$, i.e., $v=\hat{W} u+2 \beta$, where $u$ and $\beta$ are integral vectors and $u \not \equiv 0(\bmod 2)$. Thus we have

$$
\begin{aligned}
v^{T} A^{k} v & =(\hat{W} u+2 \beta)^{T} A^{k}(\hat{W} u+2 \beta) \\
& =u^{T} \hat{W}^{T} A^{k} \hat{W} u+4 u^{T} \hat{W}^{T} A^{k} \beta+4 \beta^{T} A^{k} \beta \\
& \equiv u^{T} \hat{W}^{T} A^{k} \hat{W} u \\
& \equiv 0(\bmod 4) .
\end{aligned}
$$

First, we prove the case that $n$ is even. Note that

$$
\hat{W}^{T} A^{k} \hat{W}=\left[\begin{array}{cccc}
\frac{e^{T} A^{4+k} e}{4} & \frac{e^{T} A^{5+k} e}{4} & \ldots & \frac{e^{T} A^{n / 2+2+k} e}{4} \\
\frac{e^{T} A^{5+k} e}{4} & \frac{e^{T} A^{6+k} e}{4} & \ldots & \frac{e^{T} A^{n / 2+3+k} e}{4} \\
\vdots & \vdots & \ddots & \vdots \\
\frac{e^{T} A^{n / 2+2+k} e}{4} & \frac{e^{T} A^{n / 2+3+k} e}{4} & \ldots & \frac{e^{T} A^{n+k} e}{4}
\end{array}\right] \equiv 0(\bmod 2) .
$$

Let $M=\hat{W}^{T} A^{k} \hat{W}, u=\left(u_{1}, u_{2}, \ldots, u_{l}\right)^{T}\left(l=\frac{n-2}{2}\right)$. Then it follows that

$$
\begin{aligned}
u^{T} \hat{W}^{T} A^{k} \hat{W} u & =\sum_{1 \leqslant i, j \leqslant l} M_{i j} u_{i} u_{j} \\
& =\sum_{1 \leqslant i \leqslant l} M_{i i} u_{i}^{2}+2 \sum_{1 \leqslant i<j \leqslant l} M_{i j} u_{i} u_{j} \\
& \equiv \frac{e^{T} A^{4+k} e}{4} u_{1}+\frac{e^{T} A^{6+k} e}{4} u_{2}+\ldots+\frac{e^{T} A^{n+k} e}{4} u_{l} \\
& =\left[\frac{e^{T} A^{4+k} e}{4}, \frac{e^{T} A^{6+k} e}{4}, \ldots, \frac{e^{T} A^{n+k} e}{4}\right] u \\
& \equiv 0(\bmod 4),
\end{aligned}
$$

for $k=0,1, \ldots, n-1$, where we have used Lemma 14 and $u_{i}^{2} \equiv u_{i}(\bmod 2)$ for $i=$ $1,2, \cdots, l$.

It follows from above equation that $\left[\frac{e^{T} A^{4+k} e}{4}, \frac{e^{T} A^{6+k} e}{4}, \ldots, \frac{e^{T} A^{n+k} e}{4}\right] u=4 q$ for some integer $q$. By Lemma $14, e^{T} A^{k} e \equiv 0(\bmod 8)$, for any integer $k \geqslant 3$. Thus, we have $\left[\frac{e^{T} A^{4+k} e}{8}, \frac{e^{T} A^{6+k} e}{8}, \ldots, \frac{e^{T} A^{n+k} e}{8}\right] u=2 q$, i.e.,

$$
\left[\frac{e^{T} A^{4+k} e}{8}, \frac{e^{T} A^{6+k} e}{8}, \ldots, \frac{e^{T} A^{n+k} e}{8}\right] u \equiv 0(\bmod 2)
$$

for $k=0,1, \ldots, n-1$.

Define 


$$
\begin{aligned}
& M_{1}:=\left[\begin{array}{ccccc}
\frac{e^{T} A^{4} e}{8} & \frac{e^{T} A^{6} e}{8} & \frac{e^{T} A^{8} e}{8} & \ldots & \frac{e^{T} A^{n} e}{8} \\
\frac{e^{T} A^{5} e}{8} & \frac{e^{T} A^{7} e}{8} & \frac{e^{T} A^{9} e}{8} & \ldots & \frac{e^{T} A^{n+1} e}{8} \\
\frac{e^{T} A^{6} e}{8} & \frac{e^{T} A^{8} e}{8} & \frac{e^{T} A^{10} e}{8} & \ldots & \frac{e^{T} A^{n+2} e}{8} \\
\vdots & \vdots & \vdots & \ddots & \vdots \\
\frac{e^{T} A^{n+3} e}{8} & \frac{e^{T} A^{n+5} e}{8} & \frac{e^{T} A^{n+7} e}{8} & \ldots & \frac{e^{T} A^{2 n-1} e}{8}
\end{array}\right] \\
& =\frac{1}{2}\left[\begin{array}{c}
\frac{e^{T} A}{2} \\
\frac{e^{T} A^{2}}{2} \\
\vdots \\
\frac{e^{T} A^{n-1}}{2} \\
\frac{e^{T} A^{n}}{2}
\end{array}\right]\left[\begin{array}{llll}
\frac{A^{3} e}{2} & \frac{A^{5} e}{2} & \cdots & \frac{A^{n-1} e}{2}
\end{array}\right] \\
& \equiv \frac{1}{2}\left[\begin{array}{c}
\frac{e^{T} A}{2} \\
\frac{e^{T} A^{2}}{2} \\
\vdots \\
\frac{e^{T} A^{n-1}}{2} \\
-\frac{e^{T} A^{n}}{2}-\frac{c_{n}}{2} e^{T}+e^{T}
\end{array}\right]\left[\begin{array}{llll}
\frac{A^{3} e}{2} & \frac{A^{5} e}{2} & \cdots & \frac{A^{n-1} e}{2}
\end{array}\right] \\
& =\frac{\tilde{W}^{T} \hat{W}_{1}}{2}(\bmod 2) \text {. }
\end{aligned}
$$

Then $M_{1}$ is an integral matrix and we have $M_{1} u \equiv 0(\bmod 2)$, i.e.,

$$
\frac{\tilde{W}^{T} \hat{W}_{1}}{2} u \equiv 0(\bmod 2)
$$

However, by Lemma 22, $\operatorname{rank}_{2}\left(\frac{\tilde{W}^{T} \hat{W}_{1}}{2}\right)=\left\lfloor\frac{n-1}{2}\right\rfloor$ and hence, $\frac{\bar{W}^{T} \hat{W}_{1}}{2}$ has full column rank. It follows that $u \equiv 0(\bmod 2)$. Therefore $v=\hat{W} u+2 \beta \equiv 0(\bmod 2)$, which contradicts the fact that $v \not \equiv 0(\bmod 2)$.

Next, we prove the case that $n$ is odd. According to the different definition of $\hat{W}$ (resp. $\hat{W}_{1}$ ) when $n$ is odd, we further distinguish the following two cases.

Case 1. $e^{T} A e \equiv 0(\bmod 4)$. By Lemma 15 , we have $e^{T} A^{2} e \equiv 0(\bmod 8)$. Note that

$$
\hat{W}^{T} A^{k} \hat{W}=\left[\begin{array}{cccc}
\frac{e^{T} A^{2+k} e}{4} & \frac{e^{T} A^{3+k} e}{4} & \ldots & \frac{e^{T} A^{\frac{n+1}{2}+k} e}{4} \\
\frac{e^{T} A^{3+k} e}{4} & \frac{e^{T} A^{4+k} e}{4} & \ldots & \frac{e^{T} A^{\frac{n+3}{2}+k} e}{4} \\
\vdots & \vdots & \ddots & \vdots \\
\frac{e^{T} A^{\frac{n+1}{2}+k} e}{4} & \frac{e^{T} A^{\frac{n+3}{2}+k} e}{4} & \ldots & \frac{e^{T} A^{n-1+k} e}{4}
\end{array}\right] \equiv 0(\bmod 2)
$$

Let $M=\hat{W}^{T} A^{k} \hat{W}, u=\left(u_{1}, u_{2}, \ldots, u_{l}\right)^{T}\left(l=\frac{n-1}{2}\right)$. Using the same arguments as $n$ is 
even, it follows that

$$
\begin{aligned}
u^{T} \hat{W}^{T} A^{k} \hat{W} u & \equiv\left[\frac{e^{T} A^{2+k} e}{4}, \frac{e^{T} A^{4+k} e}{4}, \ldots, \frac{e^{T} A^{n-1+k} e}{4}\right] u \\
& \equiv 0(\bmod 4),
\end{aligned}
$$

for $k=0,1, \ldots, n-1$, where we have used Lemma 14 and $u_{i}^{2} \equiv u_{i}$ for $i=1,2, \cdots, l$.

It follows from above equation that $\left[\frac{e^{T} A^{2+k} e}{4}, \frac{e^{T} A^{4+k} e}{4}, \ldots, \frac{e^{T} A^{n-1+k} e}{4}\right] u=4 q^{\prime}$ for some integer $q^{\prime}$. By Lemma $14, e^{T} A^{k} e \equiv 0(\bmod 8)$, for any integer $k \geqslant 3$. Moreover, by Lemma 15 , we have $e^{T} A^{2} e \equiv 0(\bmod 8)$. Thus, we have $\left[\frac{e^{T} A^{2+k} e}{8}, \frac{e^{T} A^{4+k} e}{8}, \ldots, \frac{e^{T} A^{n-1+k} e}{8}\right] u=$ $2 q^{\prime}$, i.e.,

$$
\left[\frac{e^{T} A^{2+k} e}{8}, \frac{e^{T} A^{4+k} e}{8}, \ldots, \frac{e^{T} A^{n-1+k} e}{8}\right] u \equiv 0(\bmod 2)
$$

for $k=0,1, \ldots, n-1$.

Define

$$
M_{2}:=\left[\begin{array}{ccccc}
\frac{e^{T} A^{2} e}{8} & \frac{e^{T} A^{4} e}{8} & \frac{e^{T} A^{6} e}{8} & \ldots & \frac{e^{T} A^{n-1} e}{8} \\
\frac{e^{T} A^{3} e}{8} & \frac{e^{T} A^{5} e}{8} & \frac{e^{T} A^{7} e}{8} & \ldots & \frac{e^{T} A^{n} e}{8} \\
\frac{e^{T} A^{4} e}{8} & \frac{e^{T} A^{6} e}{8} & \frac{e^{T} A^{8} e}{8} & \ldots & \frac{e^{T} A^{n+1} e}{8} \\
\vdots & \vdots & \vdots & \ddots & \vdots \\
\frac{e^{T} A^{n+1} e}{8} & \frac{e^{T} A^{n+3} e}{8} & \frac{e^{T} A^{n+5} e}{8} & \ldots & \frac{e^{T} A^{2 n-2} e}{8}
\end{array}\right]
$$

Then $M_{2}$ is an integral matrix and $M_{2} u \equiv 0(\bmod 2)$.

Moreover, define

$$
\begin{aligned}
M_{3}:= & {\left[\begin{array}{ccccc}
0 & 0 & 0 & \ldots & 0 \\
\frac{e^{T} A^{3} e}{8} & \frac{e^{T} A^{5} e}{8} & \frac{e^{T} A^{T} e}{8} & \ldots & \frac{e^{T} A^{n} e}{8} \\
\frac{e^{T} A^{4} e}{8} & \frac{e^{T} A^{6} e}{8} & \frac{e^{T} A^{8} e}{8} & \ldots & \frac{e^{T} A^{n+1} e}{8} \\
\vdots & \vdots & \vdots & \ddots & \vdots \\
\frac{e^{T} A^{n+1} e}{8} & \frac{e^{T} A^{n+3} e}{8} & \frac{e^{T} A^{n+5} e}{8} & \ldots & \frac{e^{T} A^{2 n-2} e}{8}
\end{array}\right] } \\
& \equiv\left[\begin{array}{ccccc}
\frac{e^{T} A^{2} e}{4} & \frac{e^{T} A^{4} e}{4} & \frac{e^{T} A^{6} e}{4} & \ldots & \frac{e^{T} A^{n-1} e}{4} \\
\frac{e^{T} A^{3} e}{8} & \frac{e^{T} A^{5} e}{8} & \frac{e^{T} A^{7} e}{8} & \ldots & \frac{e^{T} A^{n} e}{8} \\
\frac{e^{T} A^{4} e}{8} & \frac{e^{T} A^{6} e}{8} & \frac{e^{T} A^{8} e}{8} & \ldots & \frac{e^{T} A^{n+1} e}{8} \\
\vdots & \vdots & \vdots & \ddots & \vdots \\
\frac{e^{T} A^{n+1} e}{8} & \frac{e^{T} A^{n+3} e}{8} & \frac{e^{T} A^{n+5} e}{8} & \ldots & \frac{e^{T} A^{2 n-2} e}{8}
\end{array}\right] \\
= & \frac{\bar{W}^{T} \hat{W}_{1}(\bmod 2) .}{2}
\end{aligned}
$$

According to Lemma $22, \operatorname{rank}_{2}\left(M_{3}\right)=\operatorname{rank}_{2}\left(\frac{\bar{W}^{T} \hat{W}_{1}}{2}\right)=\frac{n-1}{2}$, i.e., $M_{3}$ has full column rank over $\mathbb{F}_{2}$. Comparing $M_{2}$ and $M_{3}$, it is easy to see that $\operatorname{rank}_{2}\left(M_{2}\right)=\operatorname{rank}_{2}\left(M_{3}\right)$. Then it follows from $M_{2} u \equiv 0(\bmod 2)$ that $u \equiv 0(\bmod 2)$. By the relation $v=\hat{W} u+2 \beta$, we 
get $v \equiv 0(\bmod 2)$, which contradicts the fact that $v \not \equiv 0(\bmod 2)$.

Case 2. $e^{T} A e \equiv 2(\bmod 4)$. By Lemma 15 , we have $e^{T} A^{2} e \equiv 4(\bmod 8)$. Note that

$$
\begin{aligned}
\hat{W}^{T} A^{k} \hat{W} & =\left[\begin{array}{cccc}
\frac{e^{T} A^{2+k} e}{4}-e^{T} A^{1+k} e+e^{T} A^{k} e & \frac{e^{T} A^{3+k} e}{4}-\frac{e^{T} A^{2+k} e}{2} & \ldots & \frac{e^{T} A^{\frac{n+1}{2}+k} e}{4}-\frac{e^{T} A^{\frac{n-1}{2}+k} e}{2} \\
\frac{e^{T} A^{3+k} e}{4}-\frac{e^{T} A^{2+k} e}{2} & \frac{e^{T} A^{4+k} e}{4} & \ldots & \frac{e^{T} A^{\frac{n+3}{2}+k} e}{4} \\
\vdots & \vdots & \ddots & \vdots \\
\frac{e^{T} A^{\frac{n+1}{2}+k} e}{4}-\frac{e^{T} A^{\frac{n-1}{2}+k}}{2} & \frac{e^{T} A^{\frac{n+3}{2}+k} e}{4} & \ldots & \frac{e^{T} A^{n-1+k} e}{4}
\end{array}\right] \\
& \equiv 0(\bmod 2) .
\end{aligned}
$$

Let $M=\hat{W}^{T} A^{k} \hat{W}, u=\left(u_{1}, u_{2}, \ldots, u_{l}\right)^{T}\left(l=\frac{n-1}{2}\right)$. Using the same arguments as the case $n$ is even, it follows that

$$
\begin{aligned}
u^{T} \hat{W}^{T} A^{k} \hat{W} u & \equiv\left[\frac{e^{T} A^{2+k} e}{4}-e^{T} A^{1+k}+e^{T} A^{k} e, \frac{e^{T} A^{4+k} e}{4}, \ldots, \frac{e^{T} A^{n-1+k} e}{4}\right] u \\
& \equiv 0(\bmod 4),
\end{aligned}
$$

for $k=0,1, \ldots, n-1$, or equivalently,

$$
\left[\frac{e^{T} A^{2+k} e-4 e^{T} A^{1+k} e+4 e^{T} A^{k} e}{8}, \frac{e^{T} A^{4+k} e}{8}, \ldots, \frac{e^{T} A^{n-1+k} e}{8}\right] u \equiv 0(\bmod 2)
$$

for $k=0,1, \ldots, n-1$, where we have used Lemma 14 .

Define

$$
M_{4}:=\left[\begin{array}{ccccc}
\frac{e^{T} A^{2} e-4 e^{T} A e+4 e^{T} e}{8} & \frac{e^{T} A^{4} e}{8} & \frac{e^{T} A^{6} e}{8} & \ldots & \frac{e^{T} A^{n-1} e}{8} \\
\frac{e^{T} A^{3} e-4 e^{T} A^{2} e+4 e^{T} A e}{8} & \frac{e^{T} A^{5} e}{8} & \frac{e^{T} A^{7} e}{8} & \ldots & \frac{e^{T} A^{n} e}{8} \\
\frac{e^{T} A^{4} e-4 e^{T} A^{3} e+4 e^{T} A^{2} e}{8} & \frac{e^{T} A^{6} e}{8} & \frac{e^{T} A^{8} e}{8} & \ldots & \frac{e^{T} A^{n+1} e}{8} \\
\vdots & \vdots & \vdots & \ddots & \vdots \\
\frac{e^{T} A^{n+1} e-4 e^{T} A^{n} e+4 e^{T} A^{n-1} e}{8} & \frac{e^{T} A^{n+3} e}{8} & \frac{e^{T} A^{n+5} e}{8} & \ldots & \frac{e^{T} A^{2 n-2} e}{8}
\end{array}\right] .
$$

Then $M_{4}$ is an integral matrix and we have $M_{4} u \equiv 0(\bmod 2)$.

Moreover, define

$$
\begin{array}{r}
M_{5}:=\left[\begin{array}{ccccc}
0 & 0 & 0 & \ldots & 0 \\
\frac{e^{T} A^{3} e-4 e^{T} A^{2} e+4 e^{T} A e}{8} & \frac{e^{T} A^{5} e}{8} & \frac{e^{T} A^{T} e}{8} & \ldots & \frac{e^{T} A^{n} e}{8} \\
\frac{e^{T} A^{4} e-4 e^{T} A^{3} e+4 e^{T} A^{2} e}{8} & \frac{e^{T} A^{6} e}{8} & \frac{e^{T} A^{8} e}{8} & \ldots & \frac{e^{T} A^{n+1} e}{8} \\
\vdots & \vdots & \vdots & \ddots & \vdots \\
\frac{e^{T} A^{n+1} e-4 e^{T} A^{n} e+4 e^{T} A^{n-1} e}{8} & \frac{e^{T} A^{n+3} e}{8} & \frac{e^{T} A^{n+5} e}{8} & \ldots & \frac{e^{T} A^{2 n-2} e}{8}
\end{array}\right] \\
\equiv \\
\left.\begin{array}{ccccc}
\frac{e^{T} A^{2} e-4 e^{T} A e+4 e^{T} e}{4} & \frac{e^{T} A^{4} e}{4} & \frac{e^{T} A^{6} e}{4} & \ldots & \frac{e^{T} A^{n-1} e}{4} \\
\frac{e^{T} A^{3} e-4 e^{T} A^{2} e+4 e^{T} A e}{8} & \frac{e^{T} A^{5} e}{8} & \frac{e^{T} A^{7} e}{8} & \ldots & \frac{e^{T} A^{n} e}{8} \\
\frac{e^{T} A^{4} e-4 e^{T} A^{3} e+4 e^{T} A^{2} e}{8} & \frac{e^{T} A^{6} e}{8} & \frac{e^{T} A^{8} e}{8} & \ldots & \frac{e^{T} A^{n+1} e}{8} \\
\vdots & \vdots & \vdots & \ddots & \vdots \\
\frac{e^{T} A^{n+1} e-4 e^{T} A^{n} e+4 e^{T} A^{n-1} e}{8} & \frac{e^{T} A^{n+3} e}{8} & \frac{e^{T} A^{n+5} e}{8} & \ldots & \frac{e^{T} A^{2 n-2} e}{8}
\end{array}\right]
\end{array}
$$




$$
=\frac{\bar{W}^{T} \hat{W}_{1}}{2}(\bmod 2)
$$

where in deriving the congruence relations, we have used the facts that $e^{T} A e \equiv 2(\bmod 4)$, $e^{T} A^{2} e \equiv 4(\bmod 8)$ and $e^{T} A^{k} e \equiv 0(\bmod 8)$ for $k \geqslant 3$.

According to Lemma 22, we have $\operatorname{rank}_{2}\left(M_{5}\right)=\operatorname{rank}_{2}\left(\frac{\bar{W}^{T} \hat{W}_{1}}{2}\right)=\frac{n-1}{2}$, i.e., $M_{5}$ has full column rank over $\mathbb{F}_{2}$. Comparing $M_{4}$ and $M_{5}$, we get that $\operatorname{rank}_{2}\left(M_{4}\right)=\operatorname{rank}_{2}\left(M_{5}\right)$. Then it follows from $M_{4} u \equiv 0(\bmod 2)$ that $u \equiv 0(\bmod 2)$. Thus $v=\hat{W} u+2 \beta \equiv 0(\bmod 2)$; a contradiction.

This completes the proof.

\subsection{Proof of Theorem 8 and Theorem 9}

First we present the proof of Theorem 9:

Proof. Let $W=\left[e, A e, \cdots, A^{n-1} e\right]$. Assume without loss of generality that the SNF of $W$ is $\operatorname{diag}\left(1,2^{l_{1}}, 2^{l_{2}}, \cdots, 2^{l_{n-1}} b\right)$, where $b$ is an odd square-free integer. Then there exist two unimodular matrices $U$ and $V$ such that $W=U \operatorname{diag}\left(1,2^{l_{1}}, 2^{l_{2}}, \cdots, 2^{l_{n-1}} b\right) V$ with $1 \leqslant l_{1} \leqslant l_{2} \cdots \leqslant l_{n-1}$. It follows that

$$
\begin{aligned}
U^{-1} W & =\left[U^{-1} e, U^{-1} A e, \cdots, U^{-1} A^{n-1} e\right] \\
& =\operatorname{diag}\left(1,2^{l_{1}}, 2^{l_{2}}, \cdots, 2^{l_{n-1}} b\right) V \\
& =\left[\begin{array}{cc}
1 & 0 \\
0 & 2 \Lambda
\end{array}\right]\left[\begin{array}{cc}
a & \alpha^{T} \\
\beta & V_{1}
\end{array}\right] \\
& =\left[\begin{array}{cc}
a & \alpha^{T} \\
2 \Lambda \beta & 2 \Lambda V_{1}
\end{array}\right],
\end{aligned}
$$

where $\Lambda=\operatorname{diag}\left(2^{l_{1}-1}, 2^{l_{2}-1}, \cdots, 2^{l_{n-1}-1} b\right)$ and $V=\left[\begin{array}{cc}a & \alpha^{T} \\ \beta & V_{1}\end{array}\right]$ ( $a$ is an integer, $\alpha$ and $\beta$ are column vectors of dimension $n-1$, and $V_{1}$ is a square matrix of order $n-1$ ).

It follows from $\left[U^{-1} e, U^{-1} A e, \cdots, U^{-1} A^{n-1} e\right]=\left[\begin{array}{cc}a & \alpha^{T} \\ 2 \Lambda \beta & 2 \Lambda V_{1}\end{array}\right]$ that

$$
\left[U^{-1} e, U^{-1} \frac{A e}{2}, \cdots, U^{-1} \frac{A^{n-1} e}{2}\right]=\left[\begin{array}{cc}
a & \alpha^{T} / 2 \\
2 \Lambda \beta & \Lambda V_{1}
\end{array}\right]
$$

i.e.,

$$
\bar{W}=U\left[\begin{array}{ll}
1 & 0 \\
0 & \Lambda
\end{array}\right]\left[\begin{array}{cc}
a & \alpha^{T} / 2 \\
2 \beta & V_{1}
\end{array}\right]=U\left[\begin{array}{ll}
1 & 0 \\
0 & \Lambda
\end{array}\right] V^{\prime}
$$

where $V^{\prime}:=\left[\begin{array}{cc}a & \alpha^{T} / 2 \\ 2 \beta & V_{1}\end{array}\right]$ is an integral matrix.

Note that $\operatorname{det} V^{\prime} \equiv \operatorname{det}\left[\begin{array}{cc}a & \alpha^{T} / 2 \\ 0 & V_{1}\end{array}\right] \equiv a \operatorname{det} V_{1}(\bmod 2)$. Moreover, we have

$$
\operatorname{det} V=\operatorname{det}\left[\begin{array}{cc}
a & \alpha^{T} \\
\beta & V_{1}
\end{array}\right] \equiv \operatorname{det}\left[\begin{array}{cc}
a & 0 \\
\beta & V_{1}
\end{array}\right]=a \operatorname{det} V_{1}= \pm 1(\bmod 2) \text {. }
$$


Taking determinant on both sides of Eq. (16) generates

$$
2^{\left\lfloor\frac{n-1}{2}\right\rfloor}= \pm 2^{\sum_{i=1}^{n-1}\left(l_{i}-1\right)} \operatorname{det} V^{\prime} .
$$

Note $\operatorname{det} V^{\prime}$ is odd. It is easy to see that Eq. (17) holds only if $\left\lfloor\frac{n-1}{2}\right\rfloor=\sum_{i=1}^{n-1}\left(l_{i}-1\right)$ and $\operatorname{det} V^{\prime}= \pm 1$. That is, $V^{\prime}$ is a unimodular matrix. Thus, it follows from Eq. (16) that $\left[\begin{array}{ll}1 & 0 \\ 0 & \Lambda\end{array}\right]$ is the SNF of $\bar{W}$. According to Lemma 18 we have

$$
\Lambda=\operatorname{diag}(\underbrace{1,1, \cdots, 1}_{\left\lceil\frac{n+1}{2}\right\rceil}, \underbrace{2,2, \cdots, 2 b}_{\left\lfloor\frac{n-1}{2}\right\rfloor}) .
$$

From the above equation we get immediately that the SNF of $W$ is the one given as in Eq (2), as desired.

This completes the proof.

Finally, we present the proof of Theorem 8:

Proof. Note that $G \in \Sigma_{n}$. It follows from Lemma 9 that the SNF of $W$ is of the form given as in Eq. (2), where $b$ is an odd and square-free integer. By Theorem 6 we have $\ell \mid 4 b$. Next we show $p \nmid \ell$ for any odd prime $p$. For otherwise, if $p \mid \ell$, then $p \mid b$ and $p^{2} \nmid b$ (since $b$ is odd and square-free). According to Theorem 7 we get $p \nmid \ell$; a contradiction. Therefore, we have $\ell \mid 4$. It follows from Theorem 10 that $4 \nmid \ell$, we get $\ell$ is equal to either 1 or 2.

\section{Eliminating $\ell=2$}

Let $G \in \Sigma_{n}$ and $Q \in \mathcal{Q}(G)$ with level $\ell$. In the previous section, we have established that $\ell=1$ or 2 . If the possibility that $\ell=2$ can be further eliminated, then $\ell=1$ and hence $G$ is DGS. Unfortunately, however, this is not always the case (see the example below in this section). In this section, we shall give some simple sufficient conditions for excluding the case that $\ell=2$.

First, we need the following definitions.

Definition 23. Denote $S=\left\{v \in\{0,1\}^{n} \mid v^{T} A^{k} v \equiv 0(\bmod 4)\right.$ for $\left.k=0,1, \cdots, n-1\right\}$, where $A$ is the adjacency matrix of $G$.

Definition 24. Denote $S^{\prime}=\{v \in S \mid v$ has exactly four "1" $\}$.

We have the following theorem.

Theorem 25 (c.f. Wang [15]). Let $G \in \Sigma_{n}$ and $Q \in \mathcal{Q}(G)$. If $S^{\prime}=\emptyset$, then $G$ is $D G S$.

Proof. Let $G \in \Sigma_{n}$ and $Q \in \mathcal{Q}(G)$ with the level of $\ell$. According to Theorem 8, we have $\ell=2$ or $\ell=1$. Suppose there exists a $Q \in \mathcal{Q}(G)$ with level $\ell=2$, then there must exist a column $v$ of $2 Q$ having exactly four " 1 " over $\mathbb{F}_{2}$ and hence $v \in S^{\prime}$. However, this is a contradiction since $S^{\prime}=\emptyset$. Therefore $\ell=1$ for every $Q \in \mathcal{Q}(G)$, which means that $\mathcal{Q}(G)$ contains only permutation matrices. By Theorem $3, G$ is DGS. The proof is complete. 
We remark that the condition of Theorem 25 can be efficiently verified, since we can check all $(0,1)$-vectors with exactly four " 1 " to see whether the condition $v^{T} A^{k} v \equiv$ $0(\bmod 4)$ holds for $k=0,1, \cdots, n-1$. This can be done in $O\left(n\left(\begin{array}{l}n \\ 4\end{array}\right)\right)=O\left(n^{5}\right)$ times.

The following lemma says that $S$ is closed under left-multiplication by $A$ modulo 2 .

Lemma 26. If $v \in S$, then $A v \in S$.

Proof. Note that $v \in S$, we have $v^{T} A^{k} v \equiv 0(\bmod 4)$ for $k=0,1, \cdots, n-1$. So it suffices to show that $v^{T} A^{k+2} v \equiv 0(\bmod 4)$ for $k=0,1, \cdots, n-1$. Let $P_{G}(x)=$ $x^{n}+c_{1} x^{n-1}+\cdots+c_{n-1} x+c_{n}$ be the characteristic polynomial of graph $G$. By CayleyHamilton's Theorem, we have

$$
A^{n}+c_{1} A^{n-1}+\cdots+c_{n-1} A+c_{n} I=0 .
$$

Thus we have

$$
v^{T} A^{n} v=-\left(c_{1} v^{T} A^{n-1} v+\cdots+c_{n-1} v^{T} A v+c_{n} v^{T} v\right) .
$$

So we can get $v^{T} A^{n} v \equiv 0(\bmod 4)$. Similarly we have $v^{T} A^{n+1} v \equiv 0(\bmod 4)$. This completes the proof.

Next we assume that $S^{\prime} \neq \emptyset$ (and hence $S \neq \emptyset$ ). In order to determine whether an Eulerian graph $G \in \Sigma_{n}$ is DGS, we construct a new directed graph $\Gamma=(V(\Gamma), E(\Gamma))$ associated with $G$, as follows.

- The vertex set of $\Gamma$ consists of the vectors in $S$.

- There is a directed edge from vertex $v_{i}$ to vertex $v_{j}$ if and only if $A v_{i} \equiv v_{j}(\bmod 2)$, where $v_{i}, v_{j} \in S$.

We remark that the above definition is motivated by Lemma 26, which says that the adjacency matrix $A$ acts on the set $S$, and the dynamics of its behavior can be fully captured by $\Gamma$. Also, the structural property of $\Gamma$ is closely related to the existence of a regular rational orthogonal matrix $Q \in \mathcal{Q}(G)$ with $\ell=2$, as we shall see later.

By Lemma 26, for every vector in $S$, there is exactly one outgoing edge in $\Gamma$. Note that $\Gamma$ may have loops, since we may have $A v_{i} \equiv v_{i}(\bmod 2)$ for some $i$. In particular, we always have $A \mathbf{0}=\mathbf{0}$, where $\mathbf{0} \in S$ is the zero vector.

Moreover, the directed graph $\Gamma$ constructed above exhibits simple structural characteristics. A directed cycle of $\Gamma$ is a sequence of distinct vertices $v_{1}, v_{2}, \cdots, v_{l}$ such that $v_{i} v_{i+1}$ is a directed edge of $\Gamma$ for $i=1,2, \cdots, l\left(v_{l+1}=v_{1}\right)$; it is a loop as $l=1$ and a bidirectional edge as $l=2$. It is easy to see that for any two directed cycles (including bidirectional edges and loops) $C_{1}$ and $C_{2}$ of $\Gamma$, the node sets $V\left(C_{1}\right)$ and $V\left(C_{2}\right)$ are disjoint. For any node $v \in S$, consider the trajectory, $\left\{v, A v, A^{2} v, \cdots\right\} \subset S$, of $v$. Since $S$ is finite, there exist an $n_{0}>0$ and a directed cycle $C$ such that $A^{i} v$ belongs to the cycle for $i \geqslant n_{0}$. Thus, $\Gamma$ consists of one or more components, each of which contains exactly one directed cycle.

Let us give an example for illustrations. 
Example. Let $A$ be the adjacency matrix of an Eulerian graph $G$. It can be easily computed that $\operatorname{det} W=2^{15} \times 7$. Thus we have $G \in \Sigma_{n}$. By Definition 23, we obtain the set $S$ which consists of the columns of the following matrix $\tilde{S}$ (to save space, we write the vectors in $S$ in a compact form). Then the directed graph $\Gamma=(V(\Gamma), E(\Gamma))$ can be easily constructed according to the above rules, as shown in Fig. 1.

$$
\begin{aligned}
& A=\left[\begin{array}{lllllllllll}
0 & 1 & 1 & 0 & 0 & 0 & 0 & 1 & 0 & 0 & 1 \\
1 & 0 & 0 & 1 & 0 & 0 & 1 & 0 & 0 & 1 & 0 \\
1 & 0 & 0 & 1 & 0 & 1 & 0 & 0 & 1 & 0 & 0 \\
0 & 1 & 1 & 0 & 0 & 0 & 0 & 0 & 1 & 0 & 1 \\
0 & 0 & 0 & 0 & 0 & 0 & 0 & 0 & 1 & 0 & 1 \\
0 & 0 & 1 & 0 & 0 & 0 & 0 & 1 & 0 & 1 & 1 \\
0 & 1 & 0 & 0 & 0 & 0 & 0 & 0 & 0 & 1 & 0 \\
1 & 0 & 0 & 0 & 0 & 1 & 0 & 0 & 1 & 1 & 0 \\
0 & 0 & 1 & 1 & 1 & 0 & 0 & 1 & 0 & 0 & 0 \\
0 & 1 & 0 & 0 & 0 & 1 & 1 & 1 & 0 & 0 & 0 \\
1 & 0 & 0 & 1 & 1 & 1 & 0 & 0 & 0 & 0 & 0
\end{array}\right]_{11 \times 11} \\
& \tilde{S}=\left[\begin{array}{llllllllllllllll}
0 & 0 & 0 & 0 & 0 & 0 & 0 & 0 & 1 & 1 & 1 & 1 & 1 & 1 & 1 & 1 \\
0 & 0 & 0 & 0 & 1 & 1 & 1 & 1 & 0 & 0 & 0 & 0 & 1 & 1 & 1 & 1 \\
0 & 0 & 1 & 1 & 0 & 0 & 1 & 1 & 0 & 0 & 1 & 1 & 0 & 0 & 1 & 1 \\
0 & 0 & 1 & 1 & 0 & 0 & 1 & 1 & 1 & 1 & 0 & 0 & 1 & 1 & 0 & 0 \\
0 & 0 & 0 & 0 & 1 & 1 & 1 & 1 & 0 & 0 & 0 & 0 & 1 & 1 & 1 & 1 \\
0 & 1 & 0 & 1 & 0 & 1 & 0 & 1 & 0 & 1 & 0 & 1 & 0 & 1 & 0 & 1 \\
0 & 1 & 0 & 1 & 0 & 1 & 0 & 1 & 1 & 0 & 1 & 0 & 1 & 0 & 1 & 0 \\
0 & 1 & 1 & 0 & 0 & 1 & 1 & 0 & 0 & 1 & 1 & 0 & 0 & 1 & 1 & 0 \\
0 & 0 & 0 & 0 & 1 & 1 & 1 & 1 & 0 & 0 & 0 & 0 & 1 & 1 & 1 & 1 \\
0 & 0 & 0 & 0 & 1 & 1 & 1 & 1 & 0 & 0 & 0 & 0 & 1 & 1 & 1 & 1 \\
0 & 1 & 1 & 0 & 0 & 1 & 1 & 0 & 1 & 0 & 0 & 1 & 1 & 0 & 0 & 1
\end{array}\right]_{11 \times 16}
\end{aligned}
$$

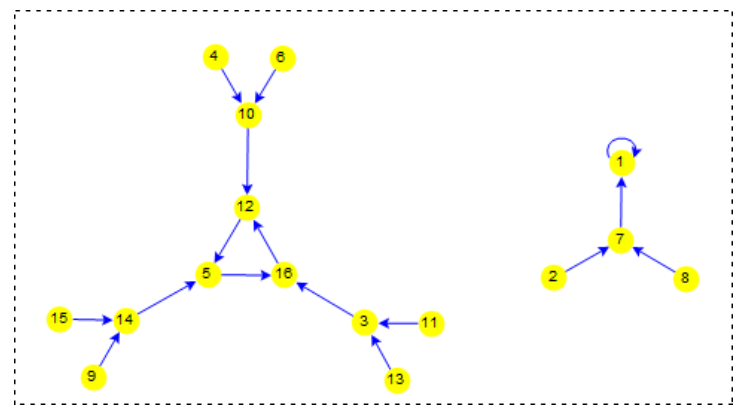

Figure 1: The directed graph $\Gamma$ constructed by the set $S$.

As it can be seen from Fig. 1, $\Gamma$ consists of two components. The left component contains a directed cycle on vertices 5, 12 and 16, and the right component contains a loop.

Besides, we remark in this example, although $G \in \Sigma_{n}$, there is a regular rational orthogonal matrix $Q \in \mathcal{Q}(G)$ with level 2 and hence, $\ell=2$ cannot be excluded. Actually, let

$$
Q=\frac{1}{2}\left[\begin{array}{ccccccccccc}
0 & 0 & 0 & 0 & -1 & 1 & 1 & 1 & 0 & 0 & 0 \\
-1 & 1 & 1 & 1 & 0 & 0 & 0 & 0 & 0 & 0 & 0 \\
0 & 0 & 0 & 0 & 1 & -1 & 1 & 1 & 0 & 0 & 0 \\
0 & 0 & 0 & 0 & 0 & 0 & 0 & 0 & 2 & 0 & 0 \\
1 & -1 & 1 & 1 & 0 & 0 & 0 & 0 & 0 & 0 & 0 \\
0 & 0 & 0 & 0 & 1 & 1 & -1 & 1 & 0 & 0 & 0 \\
0 & 0 & 0 & 0 & 0 & 0 & 0 & 0 & 0 & 2 & 0 \\
0 & 0 & 0 & 0 & 0 & 0 & 0 & 0 & 0 & 0 & 2 \\
1 & 1 & -1 & 1 & 0 & 0 & 0 & 0 & 0 & 0 & 0 \\
1 & 1 & 1 & -1 & 0 & 0 & 0 & 0 & 0 & 0 & 0 \\
0 & 0 & 0 & 0 & 1 & 1 & 1 & -1 & 0 & 0 & 0
\end{array}\right] .
$$


Then we have

$$
Q^{T} A Q=\left[\begin{array}{lllllllllll}
0 & 0 & 0 & 1 & 1 & 0 & 0 & 0 & 0 & 0 & 1 \\
0 & 0 & 1 & 0 & 0 & 0 & 0 & 1 & 1 & 1 & 1 \\
0 & 1 & 0 & 0 & 0 & 1 & 0 & 0 & 0 & 1 & 0 \\
1 & 0 & 0 & 0 & 0 & 0 & 1 & 0 & 1 & 0 & 0 \\
1 & 0 & 0 & 0 & 0 & 1 & 0 & 1 & 1 & 0 & 0 \\
0 & 0 & 1 & 0 & 1 & 0 & 1 & 0 & 0 & 0 & 1 \\
0 & 0 & 0 & 1 & 0 & 1 & 0 & 1 & 1 & 0 & 0 \\
0 & 1 & 0 & 0 & 1 & 0 & 1 & 0 & 0 & 0 & 1 \\
0 & 1 & 0 & 1 & 1 & 0 & 1 & 0 & 0 & 0 & 0 \\
0 & 1 & 1 & 0 & 0 & 0 & 0 & 0 & 0 & 0 & 0 \\
1 & 1 & 0 & 0 & 0 & 1 & 0 & 1 & 0 & 0 & 0
\end{array}\right] .
$$

Next, we show the structural property of $\Gamma$ can help us in determining whether a graph $G \in \Sigma_{n}$ is DGS.

First we need the following

Lemma 27. Let $G \in \Sigma_{n}$. Suppose there is a regular rational orthogonal matrix $Q \in \mathcal{Q}(G)$ with $\ell=2$. Let $S^{\prime \prime}:=\operatorname{span}\left\{v_{1}, v_{2}, \cdots, v_{n}\right\}$, where $v_{1}, v_{2}, \cdots, v_{n}$ are the columns of $2 Q$ over $\mathbb{F}_{2}$. Then

1. $S^{\prime \prime}$ is an A-invariant subspace;

2. $S^{\prime \prime} \subseteq S$.

Proof. It is obvious that $S^{\prime \prime}$ is a subspace of $\mathbb{F}_{2}^{n}$. Let $Q^{T} A Q=B$ for some $(0,1)$-matrix $B=\left(b_{i j}\right)_{n \times n}$. Then $A(2 Q)=(2 Q) B$. It follows that

$$
A v_{j}=b_{1 j} v_{1}+b_{2 j} v_{2}+\cdots+b_{n j} v_{n} \text { for } j=1,2, \cdots, n \text {. }
$$

Thus $A v_{j} \in S^{\prime \prime}$. So the first statement is true.

Next, in order to prove the second assertion, we only need to show $v_{i}+v_{j} \in S$ for any $v_{i} \in S^{\prime \prime}$ and any $v_{j} \in S^{\prime \prime}$. Since $v_{i} \in S^{\prime \prime}$, then $v_{i} \in S$, we get $v_{i}^{T} A^{k} v_{i} \equiv 0(\bmod 4)$. Similarly we get $v_{j}^{T} A^{k} v_{j} \equiv 0(\bmod 4)$. It follows from $Q^{T} A Q=B$ that $A^{k}(2 Q)=(2 Q) B^{k}$ for $k=0,1, \cdots, n-1$. Let $B^{k}=\left(b_{i j}^{\prime}\right)_{n \times n}$. Then we have

$$
A^{k} v_{j}=b_{1 j}^{\prime} v_{1}+b_{2 j}^{\prime} v_{2}+\cdots+b_{n j}^{\prime} v_{n} \text { for } k=0,1, \cdots, n-1
$$

Thus we have

$$
v_{i}^{T} A^{k} v_{j}=b_{1 j}^{\prime} v_{i}^{T} v_{1}+b_{2 j}^{\prime} v_{i}^{T} v_{2}+\cdots+b_{n j}^{\prime} v_{i}^{T} v_{n} \equiv 0(\bmod 2) .
$$

It follows that

$$
\left(v_{i}+v_{j}\right)^{T} A^{k}\left(v_{i}+v_{j}\right)=v_{i}^{T} A^{k} v_{i}+v_{j}^{T} A^{k} v_{j}+2 v_{i}^{T} A^{k} v_{j} \equiv 0(\bmod 4) .
$$

So the second assertion follows. This completes the proof.

Let $C_{1}, C_{2}, \cdots, C_{s}$ denote all the directed cycles of $\Gamma$ such that the corresponding component containing $C_{i}$ has at least one vector from $S^{\prime}$, for $i=1,2, \cdots, s$. Let $V_{i}:=$ $\operatorname{span}\left(C_{i}\right)$ be the vector space spanned by the nodes in $C_{i}$, for $i=1,2, \cdots, s$. Next, we give a simple condition for an Eulerian graph $G \in \Sigma_{n}$ to be DGS, by using a simple dimension argument. 
Theorem 28. Let $G \in \Sigma_{n}$. Assume that $\operatorname{dim}_{\mathbb{F}_{2}}\left(V_{i}\right)>\operatorname{dim}_{\mathbb{F}_{2}}\left(\operatorname{span}\left(S^{\prime}\right)\right)$ for $i=1,2, \cdots, s$. Then the graph $G$ is $D G S$.

Proof. Suppose $G$ is not DGS. Then there exists a $Q \in \mathcal{Q}(G)$ with level 2. Thus there is a column $v$ of $2 Q(\bmod 2)$ which belongs to $S^{\prime}$. Suppose that $A^{k} v$ is in some $C_{i}$ for sufficiently large $k$. Then $V_{i}$ is a subspace of $S^{\prime \prime}$ spanned by the column vectors of $2 Q$, according to Lemma 27 . Thus $\operatorname{dim}_{\mathbb{F}_{2}}\left(V_{i}\right) \leqslant \operatorname{dim}_{\mathbb{F}_{2}}\left(S^{\prime \prime}\right) \leqslant \operatorname{dim}_{\mathbb{F}_{2}}\left(\operatorname{span}\left(S^{\prime}\right)\right)$. This contradicts the assumption of the theorem. So for any $Q \in \mathcal{Q}(G)$, the level $\ell=1$. Thus $G$ is DGS. The proof is complete.

\section{$5 \quad$ Numerical results}

In this section, we shall give some numerical results for illustrations. All the Eulerian graphs are randomly generated using Mathematica 11.0.

First, we shall give several examples to illustrate Theorem 28.

Example 1. Let the adjacency matrix of the Eulerian graph $G$ be given as follows:

$$
A=\left[\begin{array}{llllllllllll}
0 & 1 & 1 & 0 & 0 & 1 & 1 & 0 & 0 & 0 & 0 & 0 \\
1 & 0 & 1 & 1 & 0 & 1 & 1 & 1 & 0 & 0 & 1 & 1 \\
1 & 1 & 0 & 1 & 1 & 1 & 1 & 0 & 0 & 0 & 0 & 0 \\
0 & 1 & 1 & 0 & 0 & 0 & 0 & 1 & 0 & 1 & 1 & 1 \\
0 & 0 & 1 & 0 & 0 & 1 & 1 & 0 & 0 & 1 & 0 & 0 \\
1 & 1 & 1 & 0 & 1 & 0 & 1 & 0 & 1 & 0 & 1 & 1 \\
1 & 1 & 1 & 0 & 1 & 1 & 0 & 1 & 1 & 0 & 0 & 1 \\
0 & 1 & 0 & 1 & 0 & 0 & 1 & 0 & 1 & 1 & 0 & 1 \\
0 & 0 & 0 & 0 & 0 & 1 & 1 & 1 & 0 & 1 & 1 & 1 \\
0 & 0 & 0 & 1 & 1 & 0 & 0 & 1 & 1 & 0 & 0 & 0 \\
0 & 1 & 0 & 1 & 0 & 1 & 0 & 0 & 1 & 0 & 0 & 0 \\
0 & 1 & 0 & 1 & 0 & 1 & 1 & 1 & 1 & 0 & 0 & 0
\end{array}\right]_{12 \times 12} \quad, \quad \tilde{S}=\left[\begin{array}{llllllll}
1 & 0 & 1 & 1 & 1 & 0 & 0 & 0 \\
1 & 0 & 1 & 0 & 0 & 1 & 1 & 0 \\
0 & 0 & 0 & 1 & 1 & 1 & 1 & 0 \\
0 & 0 & 0 & 1 & 1 & 1 & 1 & 0 \\
0 & 1 & 1 & 1 & 0 & 1 & 0 & 0 \\
0 & 1 & 1 & 1 & 0 & 1 & 0 & 0 \\
0 & 1 & 1 & 0 & 1 & 0 & 1 & 0 \\
0 & 1 & 1 & 0 & 1 & 0 & 1 & 0 \\
1 & 0 & 1 & 0 & 0 & 1 & 1 & 0 \\
1 & 0 & 1 & 1 & 1 & 0 & 0 & 0 \\
0 & 0 & 0 & 1 & 1 & 1 & 1 & 0 \\
0 & 0 & 0 & 1 & 1 & 1 & 1 & 0
\end{array}\right]_{12 \times 8}
$$

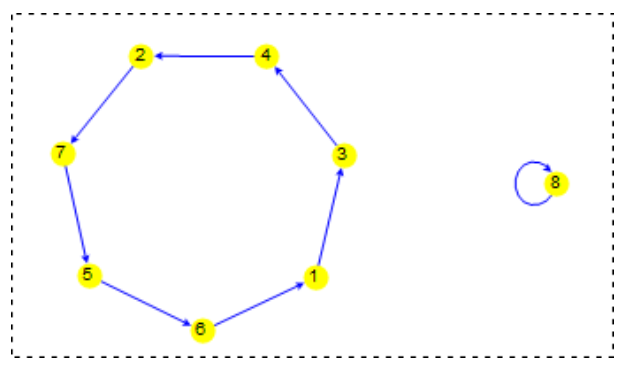

Figure 2: The directed graph $\Gamma$ constructed by the set $S$.

It can be computed easily using Mathematica 11.0 that

$$
\operatorname{det} W(G)=2^{16} \times 27925453 .
$$

Thus we have $G \in \Sigma_{n}$. The set $S$ consists of $2^{3}$ vectors over $\mathbb{F}_{2}$. The directed graph $\Gamma$ consists of exactly one directed 7-cycle and a loop, as shown in Fig. 2. Clearly the loop consisting of the zero vector can be ignored, since the set $S^{\prime}$ does not have zero vector. Denote the seven circle by $C_{1}$. Then $\operatorname{dim}_{\mathbb{F}_{2}}\left(\operatorname{span}\left(C_{1}\right)\right)=3$. However $\operatorname{dim}_{\mathbb{F}_{2}}\left(\operatorname{span}\left(S^{\prime}\right)\right)=2$, where the set $S^{\prime}=\left\{v_{1}, v_{2}\right\}$ consists of the first two columns of the matrix $\tilde{S}$. Note 
$\operatorname{dim}_{\mathbb{F}_{2}}\left(\operatorname{span}\left(S^{\prime}\right)\right)<\operatorname{dim}_{\mathbb{F}_{2}}\left(\operatorname{span}\left(C_{1}\right)\right)$. According to Theorem 28 , the graph $G$ is DGS.

Example 2. Let the adjacency matrix of the Eulerian graph $G$ be gives as follows:

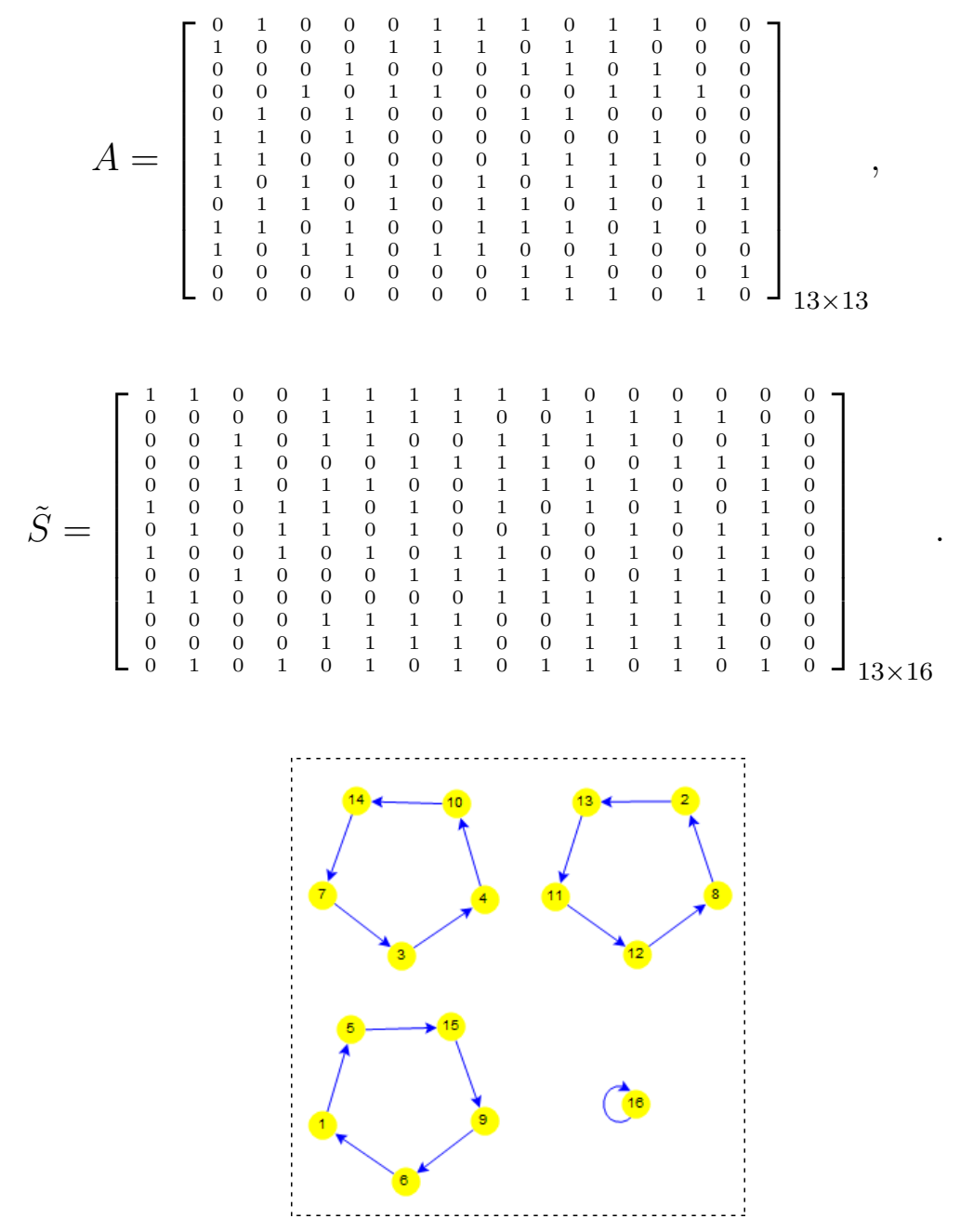

Figure 3: The directed graph $\Gamma$ constructed by the set $S$.

It can be computed easily using Mathematica 11.0 that

$$
\operatorname{det} W(G)=-2^{18} \times 7 \times 13 \times 17 \times 1185703 .
$$

Thus we have $G \in \Sigma_{n}$. Furthermore, the set $S$ consists of $2^{4}$ vectors over $\mathbb{F}_{2}$. The directed graph $\Gamma$ consists of exactly three directed 5 -cycles and a loop, as shown in Fig. 3. Ignoring the loop consisting of the zero vector, let us denote the three directed cycles by $C_{1}, C_{2}$ and $C_{3}$. Then it is easy to compute that $\operatorname{dim}_{\mathbb{F}_{2}}\left(\operatorname{span}\left(C_{i}\right)\right)=4$ for $i=1,2,3$. However, $\operatorname{dim}_{\mathbb{F}_{2}}\left(\operatorname{span}\left(S^{\prime}\right)\right)=3$, where $S^{\prime}=\left\{v_{1}, v_{2}, v_{3}, v_{4}\right\}$ consists of the first four columns of the matrix $\tilde{S}$. Since $\operatorname{dim}_{\mathbb{F}_{2}}\left(\operatorname{span}\left(S^{\prime}\right)\right)<\operatorname{dim}_{\mathbb{F}_{2}}\left(\operatorname{span}\left(C_{i}\right)\right)$ for $i=1,2,3$, according to Theorem 28 , the graph $G$ is DGS. 
Example 3. Let the adjacency matrix of the Eulerian graph $G$ be gives as follows:

$$
\begin{aligned}
& A=\left[\begin{array}{llllllllllllll}
0 & 1 & 0 & 0 & 1 & 1 & 1 & 0 & 0 & 0 & 0 & 1 & 1 & 0 \\
1 & 0 & 0 & 1 & 0 & 1 & 0 & 1 & 0 & 1 & 1 & 0 & 0 & 0 \\
0 & 0 & 0 & 1 & 0 & 1 & 1 & 0 & 1 & 0 & 1 & 0 & 0 & 1 \\
0 & 1 & 1 & 0 & 1 & 0 & 0 & 1 & 1 & 0 & 1 & 1 & 1 & 0 \\
1 & 0 & 0 & 1 & 0 & 0 & 1 & 1 & 1 & 1 & 0 & 0 & 0 & 0 \\
1 & 1 & 1 & 0 & 0 & 0 & 1 & 1 & 1 & 0 & 1 & 0 & 1 & 0 \\
1 & 0 & 1 & 0 & 1 & 1 & 0 & 1 & 1 & 0 & 0 & 0 & 1 & 1 \\
0 & 1 & 0 & 1 & 1 & 1 & 1 & 0 & 1 & 0 & 0 & 0 & 1 & 1 \\
0 & 0 & 1 & 1 & 1 & 1 & 1 & 1 & 0 & 1 & 0 & 0 & 1 & 0 \\
0 & 1 & 0 & 0 & 1 & 0 & 0 & 0 & 1 & 0 & 1 & 0 & 0 & 0 \\
0 & 1 & 1 & 1 & 0 & 1 & 0 & 0 & 0 & 1 & 0 & 0 & 1 & 0 \\
1 & 0 & 0 & 1 & 0 & 0 & 0 & 0 & 0 & 0 & 0 & 0 & 1 & 1 \\
1 & 0 & 0 & 1 & 0 & 1 & 1 & 1 & 1 & 0 & 1 & 1 & 0 & 0 \\
0 & 0 & 1 & 0 & 0 & 0 & 1 & 1 & 0 & 0 & 0 & 1 & 0 & 0
\end{array}\right]_{14 \times 14} \\
& \tilde{S}=\left[\begin{array}{llllllllllllllll}
1 & 0 & 0 & 1 & 1 & 1 & 1 & 1 & 1 & 0 & 0 & 0 & 0 & 0 & 1 & 0 \\
0 & 1 & 0 & 1 & 1 & 1 & 0 & 0 & 0 & 1 & 1 & 1 & 0 & 0 & 1 & 0 \\
1 & 0 & 0 & 1 & 1 & 1 & 1 & 1 & 1 & 0 & 0 & 0 & 0 & 0 & 1 & 0 \\
0 & 0 & 1 & 1 & 0 & 0 & 1 & 1 & 0 & 1 & 1 & 0 & 1 & 0 & 1 & 0 \\
0 & 0 & 1 & 0 & 1 & 0 & 1 & 0 & 1 & 1 & 0 & 1 & 0 & 1 & 1 & 0 \\
0 & 1 & 0 & 1 & 1 & 1 & 0 & 0 & 0 & 1 & 1 & 1 & 0 & 0 & 1 & 0 \\
0 & 1 & 0 & 0 & 0 & 1 & 0 & 1 & 1 & 1 & 0 & 0 & 1 & 1 & 1 & 0 \\
0 & 0 & 1 & 1 & 0 & 0 & 1 & 1 & 0 & 1 & 1 & 0 & 1 & 0 & 1 & 0 \\
1 & 0 & 0 & 0 & 0 & 1 & 1 & 0 & 0 & 0 & 1 & 1 & 1 & 1 & 1 & 0 \\
0 & 0 & 1 & 0 & 1 & 0 & 1 & 0 & 1 & 1 & 0 & 1 & 0 & 1 & 1 & 0 \\
1 & 0 & 0 & 0 & 0 & 1 & 1 & 0 & 0 & 0 & 1 & 1 & 1 & 1 & 1 & 0 \\
0 & 0 & 0 & 1 & 1 & 0 & 0 & 1 & 1 & 0 & 1 & 1 & 1 & 1 & 0 & 0 \\
0 & 1 & 0 & 0 & 0 & 1 & 0 & 1 & 1 & 1 & 0 & 0 & 1 & 1 & 1 & 0 \\
0 & 0 & 0 & 1 & 1 & 0 & 0 & 1 & 1 & 0 & 1 & 1 & 1 & 1 & 0 & 0
\end{array}\right]_{14 \times 16}
\end{aligned}
$$

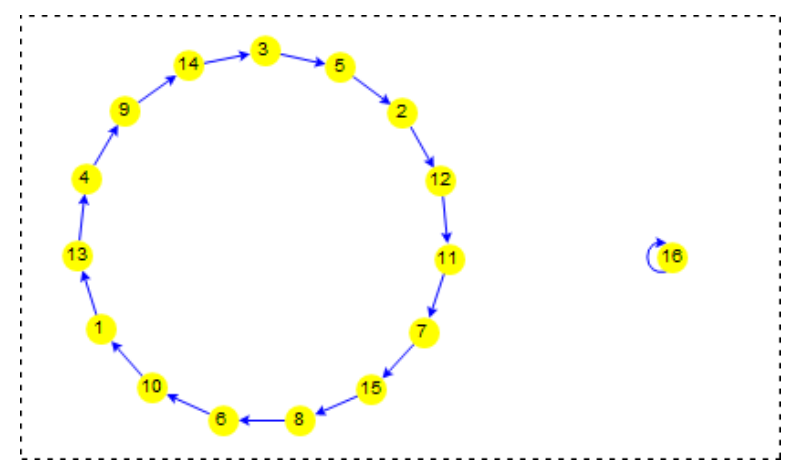

Figure 4: The directed graph $\Gamma$ constructed by the set $S$.

It can be computed easily using Mathematica 11.0 that

$$
\operatorname{det} W(G)=-2^{19} \times 3 \times 1998050003 .
$$

Thus we have $G \in \Sigma_{n}$. The set $S$ consists of $2^{4}$ vectors over $\mathbb{F}_{2}$. The directed graph $\Gamma$ consists of exactly a directed 15-cycle and a loop, as shown in Fig. 4. Ignore the loop which consists of the zero vector and denote the 15-circle by $C_{1}$, then we have $\operatorname{dim}_{\mathbb{F}_{2}}\left(\operatorname{span}\left(C_{1}\right)\right)=$ 4. However $\operatorname{dim}_{\mathbb{F}_{2}}\left(\operatorname{span}\left(S^{\prime}\right)\right)=3$, where the set $S^{\prime}=\left\{v_{1}, v_{2}, v_{3}\right\}$ consists of the first three columns of the the matrix $\tilde{S}$. Since $\operatorname{dim}_{\mathbb{F}_{2}}\left(\operatorname{span}\left(S^{\prime}\right)\right)<\operatorname{dim}_{\mathbb{F}_{2}}\left(\operatorname{span}\left(C_{1}\right)\right)$, according to Theorem 28, the graph $G$ is DGS. 
Example 4. Let the adjacency matrix of the Eulerian graph $G$ be gives as follows:

$$
A=\left[\begin{array}{lllllllllllllll}
0 & 0 & 0 & 1 & 1 & 1 & 1 & 1 & 0 & 1 & 1 & 0 & 0 & 1 & 0 \\
0 & 0 & 0 & 1 & 0 & 0 & 0 & 1 & 1 & 0 & 1 & 0 & 0 & 1 & 1 \\
0 & 0 & 0 & 0 & 0 & 0 & 0 & 0 & 0 & 1 & 1 & 1 & 1 & 1 & 1 \\
1 & 1 & 0 & 0 & 0 & 0 & 1 & 1 & 0 & 0 & 1 & 1 & 0 & 0 & 0 \\
1 & 0 & 0 & 0 & 0 & 0 & 1 & 1 & 1 & 0 & 1 & 1 & 1 & 1 & 0 \\
1 & 0 & 0 & 0 & 0 & 0 & 0 & 0 & 0 & 1 & 0 & 0 & 0 & 1 & 1 \\
1 & 0 & 0 & 1 & 1 & 0 & 0 & 0 & 1 & 1 & 0 & 1 & 1 & 1 & 0 \\
1 & 1 & 0 & 1 & 1 & 0 & 0 & 0 & 0 & 1 & 0 & 0 & 1 & 0 & 0 \\
0 & 1 & 0 & 0 & 1 & 0 & 1 & 0 & 0 & 0 & 1 & 1 & 1 & 1 & 1 \\
1 & 0 & 1 & 0 & 0 & 1 & 1 & 1 & 0 & 0 & 1 & 1 & 0 & 1 & 0 \\
1 & 1 & 1 & 1 & 1 & 0 & 0 & 0 & 1 & 1 & 0 & 1 & 0 & 1 & 1 \\
0 & 0 & 1 & 1 & 1 & 0 & 1 & 0 & 1 & 1 & 1 & 0 & 1 & 1 & 1 \\
0 & 0 & 1 & 0 & 1 & 0 & 1 & 1 & 1 & 0 & 0 & 1 & 0 & 0 & 0 \\
1 & 1 & 1 & 0 & 1 & 1 & 1 & 0 & 1 & 1 & 1 & 1 & 0 & 0 & 0 \\
0 & 1 & 1 & 0 & 0 & 1 & 0 & 0 & 1 & 0 & 1 & 1 & 0 & 0 & 0
\end{array}\right]_{15 \times 15}
$$

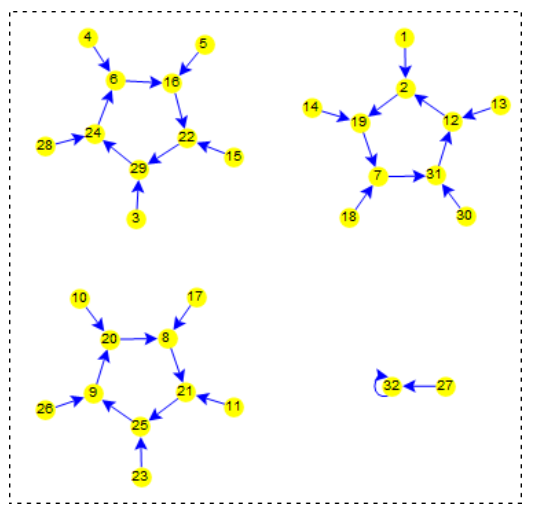

Figure 5: The directed graph $\Gamma=(V(\Gamma), E(\Gamma))$ constructed by the set $S$.

It can be computed easily using Mathematica 11.0 that

$$
\operatorname{det} W(G)=2^{21} \times 753033383825423 .
$$

Thus we have $G \in \Sigma_{n}$. Furthermore, the set $S$ consists of $2^{5}$ vectors over $\mathbb{F}_{2}$ (which are omitted to save space). The directed graph $\Gamma$ consists of exactly four components, as shown in Fig. 5. The two components in the left-bottom and right-bottom of Fig. 5 can be ignored, since the vertex set of which do not have any entry from $S^{\prime}$. Denote the two components in the left-top and right-top of Fig. 5 by $C_{i}$ for $i=1,2$. Then we have $\operatorname{dim}_{\mathbb{F}_{2}}\left(\operatorname{span}\left(C_{i}\right)\right)=4$ for $i=1,2$. However, $\operatorname{dim}_{\mathbb{F}_{2}}\left(\operatorname{span}\left(S^{\prime}\right)\right)=3$, where the set $S^{\prime}=\left\{v_{1}, v_{2}, v_{3}, v_{4}\right\}$ consists of the first four vectors in $S$. Since $\operatorname{dim}_{\mathbb{F}_{2}}\left(\operatorname{span}\left(S^{\prime}\right)\right)<$ $\operatorname{dim}_{\mathbb{F}_{2}}\left(\operatorname{span}\left(C_{i}\right)\right)$ for $i=1,2$, according to Theorem 28 , the graph $G$ is DGS.

Example 5. Let the adjacency matrix of the Eulerian graph $G$ be gives as follows:

$$
A=\left[\begin{array}{llllllllllllllll}
0 & 0 & 1 & 1 & 1 & 0 & 0 & 0 & 0 & 0 & 1 & 1 & 1 & 0 & 1 & 1 \\
0 & 0 & 1 & 0 & 0 & 1 & 0 & 1 & 1 & 1 & 1 & 1 & 0 & 1 & 1 & 1 \\
1 & 1 & 0 & 1 & 0 & 1 & 0 & 1 & 1 & 1 & 0 & 0 & 0 & 0 & 0 & 1 \\
1 & 0 & 1 & 0 & 1 & 1 & 1 & 0 & 1 & 1 & 0 & 0 & 0 & 0 & 1 & 0 \\
1 & 0 & 0 & 1 & 0 & 0 & 0 & 0 & 0 & 1 & 1 & 0 & 1 & 1 & 0 & 0 \\
0 & 1 & 1 & 1 & 0 & 0 & 1 & 1 & 1 & 0 & 1 & 0 & 0 & 1 & 1 & 1 \\
0 & 0 & 0 & 1 & 0 & 1 & 0 & 0 & 1 & 1 & 1 & 0 & 0 & 1 & 0 & 0 \\
0 & 1 & 1 & 0 & 0 & 1 & 0 & 0 & 1 & 0 & 1 & 0 & 0 & 1 & 0 & 0 \\
0 & 1 & 1 & 1 & 0 & 1 & 1 & 1 & 0 & 0 & 1 & 1 & 0 & 0 & 1 & 1 \\
0 & 1 & 1 & 1 & 1 & 0 & 1 & 0 & 0 & 0 & 0 & 0 & 1 & 0 & 1 & 1 \\
1 & 1 & 0 & 0 & 1 & 1 & 1 & 1 & 1 & 0 & 0 & 1 & 0 & 0 & 1 & 1 \\
1 & 1 & 0 & 0 & 0 & 0 & 0 & 0 & 1 & 0 & 1 & 0 & 1 & 1 & 1 & 1 \\
1 & 0 & 0 & 0 & 1 & 0 & 0 & 0 & 0 & 1 & 0 & 1 & 0 & 1 & 1 & 0 \\
0 & 1 & 0 & 0 & 1 & 1 & 1 & 1 & 0 & 0 & 0 & 1 & 1 & 0 & 1 & 0 \\
1 & 1 & 0 & 1 & 0 & 1 & 0 & 0 & 1 & 1 & 1 & 1 & 1 & 1 & 0 & 0
\end{array}\right]_{16 \times 16}
$$




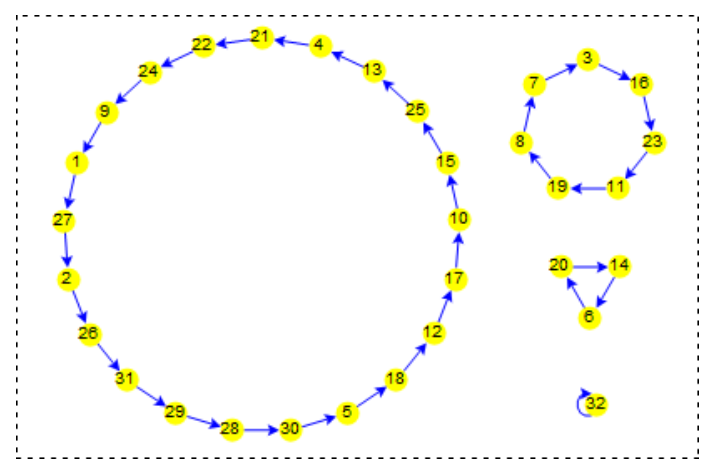

Figure 6: The directed graph $\Gamma=(V(\Gamma), E(\Gamma))$ constructed by the set $S$.

It can be computed easily using Mathematica 11.0 that

$$
\operatorname{det} W(G)=2^{22} \times 23 \times 223 \times 1559 \times 1794773 \times 51791611 .
$$

Thus we have $G \in \Sigma_{n}$. Furthermore, the set $S$ consists of $2^{5}$ vectors over $\mathbb{F}_{2}$ (which are omitted to save space). The directed graph $\Gamma$ consists of exactly four directed cycles ( namely, a 21-cycle, a 7-cycle, a 3-cycle and a loop), as shown in Fig. 6. The 7-cycle, 3 -cycle and the loop can be ignored, since the vertex sets of which do not contain any entry from $S^{\prime}$. Denote the 21-cycle by $C_{1}$, then we have $\operatorname{dim}_{\mathbb{F}_{2}}\left(\operatorname{span}\left(C_{1}\right)\right)=5$. However, $\operatorname{dim}_{\mathbb{F}_{2}}\left(\operatorname{span}\left(S^{\prime}\right)\right)=2$, where the set $S^{\prime}=\left\{v_{1}, v_{2}\right\}$ consists of the first two vectors in $S$. Since $\operatorname{dim}_{\mathbb{F}_{2}}\left(\operatorname{span}\left(S^{\prime}\right)\right)<\operatorname{dim}_{\mathbb{F}_{2}}\left(\operatorname{span}\left(C_{1}\right)\right)$, according to Theorem 28, the graph $G$ is DGS.

Finally, we have conducted a series of numerical experiments to estimate the fraction of graphs satisfying Theorem 8 and Theorem 25. The main idea is as follows:

Randomly generate $m$ Eulerian graphs of order $n(9 \leqslant n \leqslant 20)$ independently. Among these graphs, if there are $m^{\prime}$ graphs that belongs to $\Sigma_{n}$, then the fraction of graphs in $\Sigma_{n}$ among Eulerian graphs is roughly $\frac{m^{\prime}}{m}$; if there are $m^{\prime \prime}$ graph in $\Sigma_{n}$ which satisfies $S^{\prime}=\emptyset$, then the probability that a graph is DGS in $\Sigma_{n}$ is roughly $\frac{m^{\prime \prime}}{m^{\prime}}$.

Set $m=10000$, Table 1 displays the result of one of such numerical experiments.

It can be observed from Table 1, there are around $21 \%$ Eulerian graphs belong to $\Sigma_{n}$ when $n$ is odd, and the ratio is about $11 \%$ when $n$ is even. That is, the ratio for even $n$ is almost half of that for odd $n$; it would be an interesting future work to give an explanation of this phenomena.

In addition, the proportion of graphs in $\Sigma_{n}$ satisfying Theorem 25 (and hence are DGS) approaches 1 as $n$ increases, which suggests the following

Conjecture 29. Almost all graphs in $\Sigma_{n}$ are DGS.

\section{Conclusions and future work}

In this paper, we have investigated the generalized spectral characterizations of a large family of Eulerian graphs $\Sigma_{n}$, in which for every graph $G$, the power of 2 in the prime 


\begin{tabular}{ccc} 
Table 1: & Fraction of DGS graphs in \\
\hline$n$ & $\frac{m^{\prime}}{m}$ & $\frac{m^{\prime \prime}}{m^{\prime}}$ \\
\hline 9 & 0.2021 & 0.5591 \\
10 & 0.1141 & 0.6968 \\
11 & 0.2272 & 0.7025 \\
12 & 0.1120 & 0.7464 \\
13 & 0.1955 & 0.7770 \\
14 & 0.1152 & 0.7960 \\
15 & 0.2159 & 0.8425 \\
16 & 0.1086 & 0.8720 \\
17 & 0.2137 & 0.9031 \\
18 & 0.1085 & 0.9310 \\
19 & 0.2085 & 0.9535 \\
20 & 0.1117 & 0.9642 \\
\hline
\end{tabular}

factorization of det $W(G)$ is high. Thus the existing method does not work. We first show that the level of every $Q \in \mathcal{Q}(G)$ is either 1 or 2 . Then we present a simple method to eliminate the possibility that $\ell=2$, by the means of constructing a digraph associated with $G$ and then using a simple dimension argument. Numerical experiments have also been presented to illustrate the proposed method.

Besides Eulerian graphs, we mention that there are many other families of graphs for which Theorem 1.1 does not work, e.g.,

- For graphs with the degrees of all vertices being odd (of course the order of these graphs are even), Theorem 1.1 fails. This is because, for such graphs $G$, all the entries of $W(G)$ are odd and hence $\operatorname{det} W(G)$ is divisible by $2^{n-1}$;

- For all regular graphs $G$, Theorem 1.1 fails, since $\operatorname{det} W(G)$ always vanishes;

- For graphs $G$ obtained through some graph operations (such as Cartesian product, tensor product), the power of 2 in the prime factorization of $\operatorname{det} W(G)$ is usually high, although this is less obvious.

As a future work, we would like to investigate the extent to which Theorem 1.1 can be generalized, and find more families of graphs that are determined by their generalized spectra.

\section{References}

[1] L. Babai, Graph isomorphism in quasipolynomial time, Proceedings of the 48th Annual ACM SIGACT Symposium on Theory of Computing, ACM, 2016: 684-697.

[2] D.M. Cvetković, P. Rowlinson, S.K. Simić, An introduction to the Theory of Graph Spectra, Cambridge University Press, Cambridge, 2010. 
[3] H. Cohen, A Course in Computational Algebraic Number Theory, Graduate Texts in Math., Springer-Verlag, Berlin, 1993.

[4] E.R. van Dam, W.H. Haemers, Which graphs are determined by their spectrum? Linear Algebra Appl., 373 (2003) 241-272.

[5] E.R. van Dam, W.H. Haemers, Developments on spectral characterizations of graphs, Discrete Math., 309 (2009) 576-586.

[6] M. Fisher, On hearing the shape of a drum, J. Combin. Theory, 1 (1) (1966) 105-125.

[7] C.D. Godsil, Controllable Subsets in Graphs, Annals of Combinatorics, 16(4)(2012) 733-744.

[8] Hs.H. Günthard, H. Primas, Zusammenhang von Graphentheorie und MO-Theorie von Molekeln mit Systemen konjugierter Bindungen, Helv. Chim. Acta, 39 (1956) 1645-1653.

[9] E. Hückel, Quantentheoretische beitrage zum benzolproblem, Z. Phys., 70(3) (1931) 204- 286.

[10] M. Kac, Can one hear the shape of a drum? Amer. Math. Monthly, 73(4) (1966) $1-23$.

[11] S. O'Rourke, B. Touri, On a conjecture of Godsil concerning controllable random graphs, SIAM Journal on Control and Optimization, 54 (2017) 3347-3378

[12] A. Schrijver, The Theory of Linear and Integer Programming, John Wiley \& Sons, 1998.

[13] W. Wang, C.X. Xu, A sufficient condition for a family of graphs being determined by their generalized spectra, European J. Combin., 27 (2006) 826-840.

[14] W. Wang, C.X. Xu, An excluding algorithm for testing whether a family of graphs are determined by their generalized spectra, Linear Algebra Appl., 418 (2006) 62-74.

[15] W. Wang, Generalized spectral characterization revisited, The Electronic J. Combin., 20 (4) (2013), \#P4.

[16] W. Wang, A simple arithmetic criterion for graphs being determined by their generalized spectra, J. Combin. Theory, Ser. B, 122 (2017) 438-451. 